\title{
WELL-POSEDNESS OF HIGHER ORDER ABSTRACT CAUCHY PROBLEMS ${ }^{1}$
}

\author{
FRANK NEUBRANDER
}

ABStraCt. The paper is concerned with differential equations of the type

(*)

$$
u^{(n+1)}(t)-A u^{(n)}(t)-B_{1} u^{(n-1)}(t)-\cdots-B_{n} u(t)=0
$$

in a Banach space $E$ where $A$ is a linear operator with dense domain $D(A)$ and $B_{1}, \ldots, B_{n}$ are closed linear operators with $D(A) \subset D\left(B_{k}\right)$ for $1 \leq k \leq n$. The main result is the equivalence of the following two statements: (a) $A$ has nonempty resolvent set and for every initial value $\left(x_{0}, \ldots, x_{n}\right) \in(D(A))^{n+1}$ the equation (*) has a unique solution in $C^{n+1}\left(\mathbf{R}^{+}, E\right) \cap C^{n}\left(\mathbf{R}^{n},[D(A)]\right)$ ( $[D(A)]$ denotes the Banach space $D(A)$ endowed with the graph norm); (b) $A$ is the generator of a strongly continuous semigroup. Under additional assumptions on the operators $B_{k}$, which are frequently fulfilled in applications, we obtain continuous dependence of the solutions on the initial data; i.e., well-posedness of $(*)$. Using Laplace transform methods, we give explicit expressions for the solutions in terms of the operators $A, B_{k}$. The results are then used to discuss strongly damped semilinear second order equations.

0. Introduction. Of concern is the abstract Cauchy problem

$\left(\mathrm{ACP}_{n+1}\right)$

$$
\begin{aligned}
& u^{(n+1)}(t)-A u^{(n)}(t)-\sum_{k=1}^{n} B_{k} u^{(n-k)}(t)=0, \\
& u^{(j)}(0)=x_{j} \quad \text { for } 0 \leq j \leq n,
\end{aligned}
$$

where $A$ is a linear operator on a Banach space $E$ with dense domain $D(A)$ and nonempty resolvent set $\rho(A)$, and $B_{1}, B_{2}, \ldots, B_{n}$ are closed linear operators with $D(A) \subset D\left(B_{k}\right)$ for $1 \leq k \leq n$.

A function $v(\cdot) \in C^{n+1}\left(\mathbf{R}^{+}, E\right)$ is said to be a solution of $\left(\operatorname{ACP}_{n+1}\right)$ if $v^{(n-k)}(t)$ $\in D\left(B_{k}\right)$ for every $t \geq 0,0 \leq k \leq n\left(B_{0}:=A\right)$ and $\left(\mathrm{ACP}_{n+1}\right)$ is satisfied.

If $\left(\mathrm{ACP}_{n+1}\right)$ has a solution for every initial value $\bar{x}:=\left(x_{0}, x_{1}, \ldots, x_{n}\right) \in D$, where $D$ is a dense subset of $E^{n+1}$, then $\left(\mathrm{ACP}_{n+1}\right)$ is said to be solvable for a dense set of initial data.

Concerning $\left(\mathrm{ACP}_{n+1}\right)$ the following four questions will be studied in this paper:

(1) Is $\left(\mathrm{ACP}_{n+1}\right)$ solvable for a dense set of initial data?

(2) Does it only have one solution for every initial value?

(3) Can we find an explicit representation for these solutions?

(4) How do the solutions depend on their initial data?

The Cauchy problem $\left(\mathrm{ACP}_{n+1}\right)$ is called well-posed if it has unique solutions $u(\cdot)$ for a dense set of initial data which depend continuously on the initial data;

Received by the editors January 30, 1985 and, in revised form, May 2, 1985.

1980 Mathematics Subject Classification. Primary 34G10, 42D05.

Key words and phrases. Semigroups of operators, abstract Cauchy problem.

${ }^{1}$ This paper contains results of the author's dissertation, which was written at the University of Tuebingen, West Germany. 
i.e., $\lim _{n \rightarrow \infty} u_{n}^{(j)}(0)=0$ for $0 \leq j \leq n$ implies $\lim _{n \rightarrow \infty} u_{n}(t)=0$ uniformly on compact subsets of $\mathbf{R}^{+}$

After introducing some notations in $\S 1$ we will recall in $\S 2$ some results on

$$
u^{\prime}(t)-A u(t)=0, \quad u(0)=x,
$$

which was introduced as an abstract Cauchy problem for differential equations in Banach space by E. Hille in 1952 [13]. It was shown by R. S. Phillips in 1954 [23] that existence and uniqueness of solutions for every initial data in the domain of $A$ is equivalent to $A$ being the generator of a strongly continuous semigroup on $E$, assuming $A$ has a nonempty resolvent set. At the end of the second section a modified version of this theorem and a new characterization of a semigroup generator are presented.

In $\S 3$ these characterizations yield a condition on $A$ such that $\left(\mathrm{ACP}_{n+1}\right)$ is solvable for a dense set of initial data. Our main result is the following

THEOREM. Let $A$ be a densely defined linear operator and let $B_{1}, B_{2}, \ldots, B_{n}$ be closed linear operators on a Banach space $E$ with $D(A) \subset D\left(B_{k}\right)$ for $1 \leq k \leq n$. The following statements are equivalent:

(i) $A$ is the generator of a strongly continuous semigroup.

(ii) $A$ has nonempty resolvent set and for every initial value $\left(x_{0}, x_{1}, \ldots, x_{n}\right) \in$ $(D(A))^{n+1}$ the Cauchy problem $\left(\mathrm{ACP}_{n+1}\right)$ has a unique solution $u(\cdot)$ in

$$
C^{n+1}\left(\mathbf{R}^{+}, E\right) \cap C^{n}\left(\mathbf{R}^{+},[D(A)]\right)
$$

where $[D(A)]$ denotes the Banach space $D(A)$ endowed with the graph-norm $|x|_{g}:=$ $\|x\|+\|A x\|$.

For $n=0$ this theorem is exactly the Phillips characterization of a semigroup generator. Therefore it may be regarded as a generalization of Phillips' Theorem to higher order abstract Cauchy problems.

In $\S 4$ explicit expressions for the solutions in terms of the operators $A, B_{1}, B_{2}, \ldots$, $B_{n}$ are given. In $\S 5$ well-posedness results are proved under additional assumptions on the operators $B_{1}, B_{2}, \ldots, B_{n}$ which are frequently fulfilled in applications. In $\S 6$ we discuss the solvability of the Cauchy problem for

$$
u^{(2)}(t)-A u^{(1)}(t)-B u(t)=f(t, u(t)) .
$$

Among others it is shown how this damped nonlinear second order equation is related to the first order equation

$$
u^{(1)}(t)=A u(t)+\int_{0}^{t}(B u(s)+f(s, u(s)) d s .
$$

As an example, for an arbitrary semigroup generator $A$ and every initial value $(x, y) \in(D(A))^{2}$, we construct the unique solution of the inhomogeneous damped second order abstract Cauchy problem

$$
\begin{aligned}
& u^{(2)}(t)-(a A+b I) u^{(1)}(t)-(c A+d I) u(t)=f(t), \\
& u(0)=x, \quad u^{(1)}(0)=v, \quad a>0, \quad b, c, d \in \mathbf{C} .
\end{aligned}
$$

1. Notation. Let $E$ be a Banach space with norm $\|\cdot\| \cdot \sigma(A)\left(p_{\sigma}(A)\right)$ denotes the spectrum (point spectrum) and $\rho(A)(R(\lambda, A))$ the resolvent set (the resolvent) 
of a linear operator $A$ with domain $D(A) \subset E$ and range in $E$. If $A$ is a closed operator, then $\left[D\left(A^{n}\right)\right]$ denotes the Banach space

$$
\left(D\left(A^{n}\right),|x|_{n}:=\|x\|+\|A x\|+\cdots+\left\|A^{n} x\right\|\right) .
$$

By $C^{n}(I, E)$ we denote the set of all $n$-times continuously differentiable functions, by $C(I, E)$ the set of all continuous functions from an interval $I$ into $E$. For every $m \in \mathbf{N},|f|_{m}$ is defined as the following seminorm $\left(f \in C^{n}\left(\mathbf{R}^{+}, E\right)\right)$ :

$$
|f|_{m}:=\sup _{t \in[0, m]}\|f(t)\|+\sup _{t \in[0, m]}\left\|f^{(1)}(t)\right\|+\cdots+\sup _{t \in[0, m]}\left\|f^{(n)}(t)\right\| .
$$

$M_{n}$ is defined as the Fréchet space

$$
\left(C^{n}\left(\mathbf{R}^{+}, E\right),|\cdot|_{m}, m \in \mathbf{N}\right)
$$

For the elementary properties of strongly continuous semigroups we refer to $[\mathbf{2}, \mathbf{6}$, 12, 14, 16, 22].

2. Well-posedness of first order abstract Cauchy problems. We begin with a short summary of semigroup theory. A family of bounded linear operators $\{T(t), t \geq 0\}$ on a Banach space $E$ is called a strongly continuous semigroup $(T(t))$ if:

(i) $T(0)=I$

(ii) $T(t+s)=T(t) T(s)$ for $t, s \geq 0$,

(iii) $t \rightarrow T(t) x$ is continuous for every $x \in E$.

The generator $A$ of the semigroup $(T(t))$ is defined by

$$
A x=\lim _{t \rightarrow 0+} \frac{1}{t}(T(t) x-x)=T^{\prime}(0+) x
$$

whenever the limit exists. The domain $D(A)$ of $A$ is the set of all $x \in E$ for which this limit exists. $A$ is a densely defined, closed linear operator with nonempty resolvent set $\rho(A)$. For every strongly continuous semigroup $(T(t))$ there exist constants $w \in \mathbf{R}$ and $M \geq 1$ such that

$$
\|T(t)\| \leq M e^{w t} .
$$

Every $\lambda \in \mathbf{C}$ with $\operatorname{Re} \lambda>w$ is an element of the resolvent set of $A$ and for such elements of $\mathbf{C}$ the resolvent $R(\lambda, A)$ of $A$ is given as the Laplace transform of the semigroup $(T(t))$; i.e.,

$$
R(\lambda, A) x=\int_{0}^{\infty} e^{-\lambda t} T(t) x d t \quad \text { for every } x \in E \text { and every } \lambda \in \mathbf{C} \text { with } \operatorname{Re} \lambda>w .
$$

A linear operator $A$ is the generator of a strongly continuous semigroup if and only if

(i) $A$ is densely defined,

(ii) there exist $w \in \mathbf{R}$ and $M \geq 1$ such that every $\lambda \in \mathbf{C}$ with $\operatorname{Re} \lambda>w$ is an element of $\rho(A)$ and $\left\|R(\lambda, A)^{n}\right\| \leq M(\operatorname{Re} \lambda-w)^{-n}$ for every $n \in \mathbf{N}$.

This is the famous Hille-Yosida characterization of a semigroup generator.

If $A$ is the generator of a strongly continuous semigroup $(T(t))$, then, for every $x \in D(A), t \rightarrow T(t) x$ is the unique solution of the first order abstract Cauchy problem

$\left(\mathrm{ACP}_{1}\right) \quad u(t)=A u(t), \quad u(0)=x$. 
Hille [13], Phillips [23] and Fattorini [7] gave different definitions of well-posedness of $\left(\mathrm{ACP}_{1}\right)$. They showed that if $A$ is a linear operator with dense domain and nonempty resolvent set, then $\left(\mathrm{ACP}_{1}\right)$ is "well-posed" if and only if $A$ generates a strongly continuous semigroup. In the following theorem we recall their results; i.e., we compile the equivalent definitions of well-posedness of $\left(\mathrm{ACP}_{1}\right)$. For a proof see, for example, [21].

THEOREM 1. Let $A$ be a densely defined linear operator on a Banach space $E$. Consider the abstract Cauchy problem

$$
u^{\prime}(t)=A u(t), \quad u(0)=x
$$

The following statements are equivalent:

(i) $A$ is the generator of a strongly continuous semigroup.

(ii) $\rho(A) \neq \varnothing$ and for every $x \in D(A),\left(\mathrm{ACP}_{1}\right)$ has a unique solution $u(\cdot)$ in $C^{1}\left(\mathbf{R}^{+}, E\right) \cap C\left(\mathbf{R}^{+},[D(A)]\right)$ (well-posedness in the sense of Phillips).

(iii) $A$ is closed and there exists a dense subset $D$ such that, for every $x \in D$, $\left(\mathrm{ACP}_{1}\right)$ has a solution $u(\cdot): \mathbf{R}^{+} \rightarrow D(A)$ in $C^{\mathbf{1}}\left(\mathbf{R}^{+}, E\right)$. For every sequence of solutions with $u_{n}(0) \rightarrow 0$ we have $u_{n}(\cdot) \rightarrow 0$ uniformly on compacts of $\mathbf{R}^{+}$(wellposedness in the sense of Fattorini).

(iv) $A$ is closed and there exists a dense subset $D$ such that, for every $x \in D$, $\left(\mathrm{ACP}_{1}\right)$ has a solution $u(\cdot): \mathbf{R}^{+} \rightarrow D(A)$ in $C^{\mathbf{1}}\left(\mathbf{R}^{+}, E\right)$. There are constants $M, w$ such that for every solution $u(\cdot)$ and every $t \in \mathbf{R}^{+}$,

$$
\|u(t)\| \leq M e^{w t}\|u(0)\|
$$

(well-posedness in the sense of Hille).

The second theorem of this section is a modified version of the characterization of a semigroup generator by well-posedness of $\left(\mathrm{ACP}_{1}\right)$ in the sense of Phillips.

THEOREM 2. Let $A$ be a linear operator on a Banach space $E$ such that $D\left(A^{n+1}\right)$ is dense in $E$ for some $n \in \mathbf{N} \cup\{0\}$. Then the following statements are equivalent:

(i) $A$ is the generator of a strongly continuous semigroup.

(ii) $\rho(A) \neq \varnothing$ and, for every $x \in D\left(A^{n+1}\right)$, ( $\left.\mathrm{ACP}_{1}\right)$ has a unique solution $u(\cdot): \mathbf{R}^{+} \rightarrow D(A)$ in $C^{n+1}\left(\mathbf{R}^{+}, E\right)$.

PROOF. (i) $\rightarrow$ (ii) follows from elementary semigroup theory. We show: (ii) implies that $\left(\mathrm{ACP}_{1}\right)$ is well-posed in the sense of Fattorini. $A$ is densely defined with nonempty resolvent set, hence closed. For every $x \in D:=D\left(A^{n+1}\right),\left(\mathrm{ACP}_{1}\right)$ has a unique solution $u: \mathbf{R}^{+} \rightarrow D(A)$ in $C^{n+1}\left(\mathbf{R}^{+}, E\right) \subset C^{1}\left(\mathbf{R}^{+}, E\right)$. We define a linear operator $K:\left[D\left(A^{n+1}\right)\right] \rightarrow M_{n+1}$ by $x \rightarrow K x:=u(\cdot)$, where $u(\cdot)$ is the unique solution of $\left(\mathrm{ACP}_{1}\right) . K$ is a closed, everywhere defined and hence continuous linear operator.

Now let $u_{k}(\cdot)$ be a sequence of solutions of $\left(\mathrm{ACP}_{1}\right)$ such that $u_{k}(0) \rightarrow 0$ for $k \rightarrow \infty$. We will show that $u_{k}(\cdot) \rightarrow 0$ uniformly on compacts of $\mathbf{R}^{+}$(well-posedness 
in the sense of Fattorini). Define $v_{k}(\cdot):=R(\lambda, A)^{n+1} u_{k}(\cdot)$. Then $v_{k}(\cdot): \mathbf{R}^{+} \rightarrow$ $D\left(A^{n+1}\right)$ and $A^{j} v_{k}(0) \rightarrow 0$ for $k \rightarrow \infty$ and every $0 \leq j \leq n+1$. Hence $v_{k}(0) \rightarrow 0$ in $\left[D\left(A^{n+1}\right)\right]$.

Now we show by induction that $v_{k}^{(j-1)}(\cdot)$ is differentiable and that $v_{k}^{(j)}(\cdot)=$ $A^{j} v_{k}(\cdot)$ for every $1 \leq j \leq n+1$. The statement is true for $j=1$. Suppose it is true for every $1 \leq m \leq j$ where $1 \leq j \leq n$. Then it is true for $j+1$ as can be seen by

$$
(\lambda-A)^{j} v_{k}(\cdot)=R(\lambda, A)^{n+1-j} u_{k}(\cdot)
$$

or

$$
\begin{aligned}
v_{k}^{(j)}(\cdot) & =A^{j} v_{k}(\cdot) \\
& =(-1)^{j}\left[R(\lambda, A)^{n+1-j} u_{k}(\cdot)-\sum_{r=1}^{j}\left(\begin{array}{l}
j \\
r
\end{array}\right) \lambda^{r}(-1)^{j-r} v_{k}^{(j-r)}(\cdot)\right] .
\end{aligned}
$$

Therefore $v_{k}^{(j)}(\cdot)$ is continuously differentiable and

$$
\begin{aligned}
v_{k}^{(j+1)}(\cdot) & =(-1)^{j}\left[R(\lambda, A)^{n+1-j} \dot{u}_{k}(\cdot)-\sum_{r=1}^{j}\left(\begin{array}{l}
j \\
r
\end{array}\right) \lambda^{r}(-1)^{j-r} v_{k}^{(j-r+1)}(\cdot)\right] \\
& =A\left((-1)^{j}\left[R(\lambda, A)^{n+1-j} u_{k}(\cdot)-\sum_{r=1}^{j}\left(\begin{array}{l}
j \\
r
\end{array}\right) \lambda^{r}(-1)^{j-r} v_{k}^{(j-r)}(\cdot)\right]\right) \\
& =A^{j+1} v_{k}(\cdot) .
\end{aligned}
$$

We have seen that $v_{k}(\cdot)$ is a solution of $\left(\mathrm{ACP}_{1}\right)$ in $C^{n+1}\left(\mathbf{R}^{+}, E\right)$, and by the continuity of $K$ we obtain $v_{k}(\cdot)=K v_{k}(0) \rightarrow 0$ in $M_{n+1}$, which implies that $v_{k}^{(j)}(\cdot) \rightarrow 0$ uniformly on compacts of $\mathbf{R}^{+}$for every $0 \leq j \leq n+1$. But

$$
\begin{aligned}
u_{k}(\cdot) & =(\lambda-A)^{n+1} v_{k}(\cdot) \\
& =\sum_{r=0}^{n+1}\left(\begin{array}{c}
n+1 \\
r
\end{array}\right)(\lambda)^{r}(-1)^{n+1-r} v_{k}^{n+1-r}(\cdot)
\end{aligned}
$$

and therefore $u_{k}(\cdot) \rightarrow 0$ uniformly on compacts of $\mathbf{R}^{+}$.

In the subsequent sections we will give sufficient conditions for solvability and well-posedness of

$\left(\mathrm{ACP}_{n+1}\right)$

$$
\begin{aligned}
& u^{(n+1)}(t)-A u^{(n)}(t)-\sum_{k=1}^{n} B_{k} u^{(n-k)}(t)=0 \\
& u^{(j)}(0)=x_{j} \quad \text { for } 0 \leq j \leq n
\end{aligned}
$$

where $A$ is a densely defined linear operator on a Banach space $E$ with nonempty resolvent set $\rho(A)$ and $B_{1}, B_{2}, \ldots, B_{n}$ are closed linear operators with $D(A) \subset$ $D\left(B_{k}\right)$ for $1 \leq k \leq n$.

The results there depend heavily on the following characterization of a semigroup generator. In the proof we make use of the Phillips perturbation theorem (see, for example, [24]) which says that if $A$ is a semigroup generator and $B$ is a bounded linear operator, then $A+B$ is a semigroup generator too. 
THEOREM 3. Let $A$ be a densely defined linear operator and let $B_{1}, B_{2}, \ldots, B_{n}$ be closed linear operators on $E$ with $D(A) \subset D\left(B_{k}\right)$ for $1 \leq k \leq n$. Then the following statements are equivalent:

(i) $A$ is the generator of a strongly continuous semigroup on $E$.

(ii)

$$
\Delta:=\left[\begin{array}{cccccc}
A & I & 0 & \cdot & \cdot & 0 \\
B_{1} & 0 & I & \cdot & \cdot & 0 \\
B_{2} & 0 & 0 & \cdot & \cdot & 0 \\
\cdot & \cdot & \cdot & \cdot & \cdot & \cdot \\
\cdot & \cdot & \cdot & \cdot & \cdot & I \\
B_{n} & 0 & 0 & \cdot & \cdot & 0
\end{array}\right]
$$

is the generator of a strongly continuous semigroup on $E^{n+1}$.

Proof. (a) (i) $\rightarrow$ (ii). Let $\lambda \in \rho(A)$. Define $A_{0}:=(-\lambda+A)$. Then $A_{0}$ is the generator of a strongly continuous semigroup $(T(t)), 0 \in \rho\left(A_{0}\right)$ and $D\left(A_{0}\right)=D(A)$. Define

$$
\Psi:=\left[\begin{array}{cccccc}
\lambda I & I & 0 & . & . & 0 \\
0 & 0 & I & . & . & 0 \\
0 & 0 & 0 & . & . & 0 \\
. & . & . & . & . & . \\
. & . & . & . & . & I \\
0 & 0 & 0 & . & . & 0
\end{array}\right]
$$

and

$$
\Delta_{0}:=\left[\begin{array}{cccccc}
A_{0} & 0 & 0 & \cdot & \cdot & 0 \\
B_{1} & 0 & 0 & \cdot & \cdot & 0 \\
B_{2} & 0 & 0 & \cdot & \cdot & 0 \\
\cdot & \cdot & \cdot & \cdot & \cdot & \cdot \\
\cdot & \cdot & \cdot & \cdot & \cdot & \cdot \\
B_{n} & 0 & 0 & \cdot & \cdot & 0
\end{array}\right]
$$

Then $\Delta:=\Delta_{0}+\Psi$. We will see that $\Delta_{0}$ is the generator of a strongly continuous semigroup on $E^{n+1}$. Hence, by the Phillips perturbation theorem, $\Delta$ is the generator of a strongly continuous semigroup. From the closedness of the operators $B_{k}$ $(1 \leq k \leq n)$ and from $D(A) \subset D\left(B_{k}\right)$ we obtain that $B_{k} R\left(0, A_{0}\right)$ is a continuous linear operator on $E(1 \leq k \leq n)$.

For every $t \in \mathbf{R}^{+}$define a bounded linear operator $\Gamma(t)$ on $E^{n+1}$ by

$$
\Gamma(t):=\left[\begin{array}{cccccc}
T(t) & 0 & 0 & \cdot & \cdot & 0 \\
B_{1} R\left(0, A_{0}\right)(I-T(t)) & I & 0 & \cdot & \cdot & 0 \\
B_{2} R\left(0, A_{0}\right)(I-T(t)) & 0 & I & \cdot & \cdot & 0 \\
\cdot & \cdot & \cdot & \cdot & \cdot & \cdot \\
\cdot & \cdot & \cdot & \cdot & \cdot & \cdot \\
B_{n} R\left(0, A_{0}\right)(I-T(t)) & 0 & 0 & \cdot & \cdot & I
\end{array}\right] .
$$

By simple matrix calculations one shows that $(\Gamma(t))$ is a strongly continuous semigroup on $E^{n+1}$ with generator $\Delta_{0}$. 
(b) (ii) $\rightarrow$ (i). If $\Delta$ is the generator of a strongly continuous semigroup, then the same is true for $\Delta_{1}$ where

$$
\Delta_{1}:=\left[\begin{array}{cccc}
A & 0 & \cdot & 0 \\
B_{1} & 0 & \cdot & 0 \\
\cdot & \cdot & \cdot & \cdot \\
B_{n} & 0 & \cdot & 0
\end{array}\right] .
$$

It is easy to see that the resolvent set of $A$ has to be nonempty and that $R\left(\lambda, \Delta_{1}\right)^{m}$ is given by

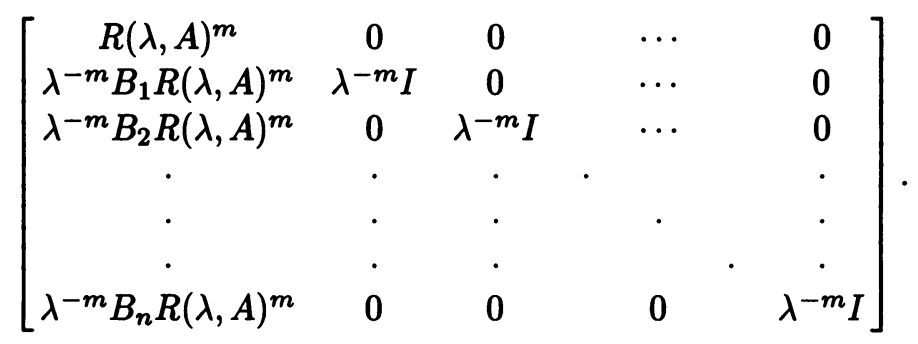

Hence we obtain for the densely defined linear operator $A$ with nonempty resolvent set that

$$
\left\|R(\lambda, A)^{m}\right\| \leq\left\|R\left(\lambda, \Delta_{1}\right)^{m}\right\| \leq M(\operatorname{Re} \lambda-w)^{-m}
$$

for every $m \in \mathbf{N}$ and every $\lambda \in \mathbf{C}$ with $\operatorname{Re} \lambda>w$. By the Hille-Yosida characterization it follows that $A$ is the generator of a strongly continuous semigroup.

If $A$ is the generator of a compact or holomorphic semigroup (for $t>0$ ) so is $A+B$ for every bounded $B$ (see, for example [24]). In the proof of Theorem 3 we have seen that the semigroup generated by

$$
\Delta_{0}:=\left[\begin{array}{cccccc}
A_{0} & 0 & 0 & \cdot & \cdot & 0 \\
B_{1} & 0 & 0 & \cdot & \cdot & 0 \\
B_{2} & 0 & 0 & \cdot & \cdot & 0 \\
\cdot & \cdot & \cdot & \cdot & \cdot & \cdot \\
\cdot & \cdot & \cdot & \cdot & \cdot & \cdot \\
B_{n} & 0 & 0 & \cdot & \cdot & 0
\end{array}\right]
$$

is given by

$$
\Gamma(t):=\left[\begin{array}{cccccc}
T(t) & 0 & 0 & \cdot & \cdot & 0 \\
B_{1} R\left(0, A_{0}\right)(I-T(t)) & I & 0 & \cdot & \cdot & 0 \\
B_{2} R\left(0, A_{0}\right)(I-T(t)) & 0 & I & \cdot & \cdot & 0 \\
\cdot & \cdot & \cdot & \cdot & \cdot & \cdot \\
\cdot & \cdot & \cdot & \cdot & \cdot & \cdot \\
B_{n} R\left(0, A_{0}\right)(I-T(t)) & 0 & 0 & \cdot & \cdot & I
\end{array}\right] .
$$

From this and the fact that $\Delta$ is a semigroup generator on $E^{n+1}$ if and only if $A$ is a semigroup generator on $E$ we obtain the following corollary.

COROLlaRY 4. Let $A$ be a densely defined linear operator and let $B_{1}, B_{2}, \ldots$, $B_{n}$ be closed linear operators on $E$ with $D(A) \subset D\left(B_{k}\right)$ for $1 \leq k \leq n$. Then the 
following statements are equivalent:

(i) $A$ is the generator of a compact or holomorphic semigroup on $E$.

(ii) $\Delta$ is the generator of a compact or holomorphic semigroup on $E^{n+1}$.

3. Solvability of higher order abstract Cauchy problems. In [9] H. O. Fattorini considered the Cauchy probelm for

$$
u^{(2)}(t)-A u^{(1)}(t)-B u(t)=0,
$$

where $A$ and $B$ are densely defined linear operators on a Banach space $E$. He studied properties of the solutions under the assumption that (\#) is solvable for a dense subset of initial data such that the solutions depend continuously on its initial data; i.e., there exist locally bounded functions $K_{0}(\cdot), K_{1}(\cdot)$ from $\mathbf{R}^{+}$into $\mathbf{R}^{+}$such that

$$
\|u(t)\| \leq K_{0}(t)\|u(0)\|+K_{1}(t)\left\|u^{(1)}(0)\right\|
$$

for every solution $u(\cdot)$ of $(\#)$. Further he wrote: "We shall not be concerned here with the problem of finding conditions on the coefficients $A, B$ in order that the Cauchy problem for (\#) be well posed in some sense or another..."

This section deals with the problem of finding conditions on $A, B_{1}, B_{2}, \ldots, B_{n}$ such that the Cauchy problem for

$$
u^{(n+1)}(t)-A u^{(n)}(t)-\sum_{k=1}^{n} B_{k} u^{(n-k)}(t)=0
$$

is solvable for a dense set of initial data.

We remark that Phillips' definition of well-posedness for $\left(\mathrm{ACP}_{1}\right)$ and statement (ii) in the following theorem agree when $n=0$. Therefore one may regard the following result as a generalization of the Phillips characterization of a semigroup generator to higher order abstract Cauchy problems. For a discussion of the continuous dependence of the solutions from the initial data, see $\S 5$.

THEOREM 5. Let $A$ be a densely defined linear operator and let $B_{1}, B_{2}, \ldots, B_{n}$ be closed linear operators on a Banach space $E$ with $D(A) \subset D\left(B_{k}\right)$ for $1 \leq k \leq n$. Consider the following Cauchy problem:

$$
\begin{gathered}
\left(\mathrm{ACP}_{n+1}\right) \quad u^{(n+1)}(t)-A u^{(n)}(t)-\sum_{k=1}^{n} B_{k} u^{(n-k)}(t)=0, \\
u^{(j)}(0)=x_{j}, \quad 0 \leq j \leq n .
\end{gathered}
$$

Then the following statements are equivalent:

(i) $A$ is the generator of a strongly continuous semigroup.

(ii) $A$ has nonempty resolvent set and for every initial value $\left(x_{0}, x_{1}, \ldots, x_{n}\right) \in$ $(D(A))^{n+1}$ the Cauchy problem $\left(\mathrm{ACP}_{n+1}\right)$ has a unique solution $u(\cdot)$ in

$$
C^{n+1}\left(\mathbf{R}^{+}, E\right) \cap C^{n}\left(\mathbf{R}^{+},[D(A)]\right) .
$$

(iii) $A$ has nonempty resolvent set and for every initial value $\left(x_{0}, x_{1}, \ldots, x_{n}\right) \in$ $(D(A))^{n+1}$ the Cauchy problem $\left(\mathrm{ACP}_{n+1}\right)$ has a solution $u(\cdot)$ in $C^{n+1}\left(\mathbf{R}^{+}, E\right)$ such that the following conditions hold.

(a) $u^{(n-k)}(\cdot): \mathbf{R}^{+} \rightarrow D\left(B_{k}\right)$ for $0 \leq k \leq n\left(B_{0}:=A\right)$,

(b) $B_{k} u^{(n-k)}(\cdot)$ are continuous for $1 \leq k \leq n$.

The solution is unique among all solutions satisfying (a) and (b). 
PrOOF. (a) (i) $\rightarrow$ (ii). The proof of this implication is divided into two main parts. In the first one (Lemma 5.1) we prove that the operator

$$
\Psi:=\left[\begin{array}{cccccc}
I & 0 & 0 & \cdot & \cdot & 0 \\
-A & I & 0 & \cdot & \cdot & 0 \\
-B_{1} & -A & I & \cdot & \cdot & 0 \\
\cdot & \cdot & \cdot & \cdot & \cdot & \cdot \\
\cdot & \cdot & \cdot & \cdot & I & 0 \\
-B_{n-1} & -B_{n-2} & \cdot & -B_{1} & -A & I
\end{array}\right]
$$

maps $D(A)^{n+1}$ onto $D\left(\Delta^{n+1}\right)$ where $\Delta$ is defined by

$$
\Delta:=\left[\begin{array}{cccccc}
A & I & 0 & \cdot & \cdot & 0 \\
B_{1} & 0 & I & \cdot & \cdot & 0 \\
B_{2} & 0 & 0 & \cdot & \cdot & 0 \\
\cdot & \cdot & \cdot & \cdot & \cdot & \cdot \\
\cdot & \cdot & \cdot & \cdot & \cdot & I \\
B_{n} & 0 & 0 & \cdot & \cdot & 0
\end{array}\right] .
$$

By Theorem $3, \Delta$ is the generator of a strongly continuous semigroup on $E^{n+1}$. Hence, by Theorem 2, the Cauchy problem

$$
u^{\prime}(t)=\Delta u(t), \quad u(0)=\Psi \bar{x}
$$

has for every $\bar{x} \in D(A)^{n+1}$ a unique solution $u(\cdot)$ in $C^{n+1}\left(\mathbf{R}^{+}, E^{n+1}\right)$. In the second part, beginning with Lemma 5.2, we show that the first coordinate of this solution is a solution of $\left(\mathrm{ACP}_{n+1}\right)$ in $C^{n+1}\left(\mathbf{R}^{+}, E\right) \cap C^{n}\left(\mathbf{R}^{+},[D(A)]\right)$ with initial value $\bar{x}:=\left(x_{0}, x_{1}, \ldots, x_{n}\right) \in D(A)^{n+1}$.

LEMMA 5.1. $\Psi$ is a bijection from $D(A)^{n+1}$ onto $D\left(\Delta^{n+1}\right)$.

PrOOF. An induction shows that $\Delta^{k} \Psi(1 \leq k \leq n)$ is given by

$$
\left[\begin{array}{cc}
0 & Y_{k} \\
Z_{k} & 0
\end{array}\right]
$$

where

$$
\begin{aligned}
& Z_{k}:=\left[\begin{array}{ccccc}
B_{n} & B_{n-1} & . & . & B_{n-k+1} \\
0 & B_{n} & \cdot & . & \cdot \\
0 & 0 & \cdot & . & . \\
\cdot & \cdot & \cdot & . & \cdot \\
\cdot & . & \cdot & B_{n} & B_{n-1} \\
0 & 0 & \cdot & 0 & B_{n}
\end{array}\right], \\
& Y_{k}:=\left[\begin{array}{cccccc}
I & 0 & 0 & \cdot & \cdot & 0 \\
-A & I & 0 & & & 0 \\
-B_{1} & -A & I & \cdot & & 0 \\
\cdot & \cdot & \cdot & \cdot & \cdot & \cdot \\
\cdot & \cdot & \cdot & \cdot & \cdot & 0 \\
-B_{n-1-k} & \cdot & \cdot & -B_{1} & -A & I
\end{array}\right],
\end{aligned}
$$




$$
Y_{n-1}:=\left[\begin{array}{cc}
I & 0 \\
-A & I
\end{array}\right], \quad Y_{n}:=I \text {. }
$$

From this we obtain $\Psi \bar{x} \in D\left(\Delta^{n+1}\right)$ for every $\bar{x}=\left(x_{0}, x_{1}, \ldots, x_{n}\right) \in D(A)^{n+1}$. It is easy to see that $\Psi$ is an injective map. By another induction one shows that $D\left(\Delta^{k}\right)$ is recursively given by

$$
\begin{aligned}
D(\Delta)= & \left\{\left(y_{0}, y_{1}, \ldots, y_{n}\right) \in E^{n+1}: x_{0}:=y_{0} \in D(A)\right\}, \\
D\left(\Delta^{k+1}\right)= & \left\{\left(y_{0}, y_{1}, \ldots, y_{n}\right) \in E^{n+1}: x_{j} \in D(A) \text { for } 0 \leq j \leq k-1\right. \\
& \text { and } \left.x_{k}:=\sum_{i+j=k-1} B_{i} x_{j}+y_{k} \in D(A)\left(B_{0}:=A\right)\right\} .
\end{aligned}
$$

Now let $\left(y_{0}, y_{1}, \ldots, y_{n}\right) \in D\left(\Delta^{n+1}\right)$. Define $x_{0}:=y_{0}$ and $x_{k}:=\sum_{i+j=k-1} B_{i} x_{j}+y_{k}$ $\left(B_{0}:=A\right)$. Then $\left(x_{0}, x_{1}, \ldots, x_{n}\right) \in D(A)^{n+1}$ and

$$
\Psi\left(x_{0}, x_{1}, \ldots, x_{n}\right)=\left(-\sum_{i+j=k-1} B_{i} x_{j}+x_{k}\right)_{0 \leq k \leq n}=\left(y_{0}, y_{1}, \ldots, y_{n}\right) .
$$

LEMMA 5.2. Let $A$ be a densely defined linear operator with nonempty resolvent set. The following statements are equivalent:

(i) $u(\cdot) \in C^{n}\left(\mathbf{R}^{+}, E\right), u(\cdot): \mathbf{R}^{+} \rightarrow D(A)$, and $A u(\cdot) \in C^{n}\left(\mathbf{R}^{+}, E\right)$.

(ii) $u(\cdot) \in C^{n}\left(\mathbf{R}^{+},[D(A)]\right)$.

(iii) $u(\cdot) \in C^{n}\left(\mathbf{R}^{+}, E\right), u(\cdot): \mathbf{R}^{+} \rightarrow D(A)$ and $B u(\cdot) \in C^{n}\left(\mathbf{R}^{+}, E\right)$ for every closed operator $B$ with $D(A) \subset D(B)$.

PROOF. A densely defined operator with nonempty resolvent set is closed. The implication (i) $\rightarrow$ (ii) follows from the definition of the Banach space $[D(A)]$. To prove the implication (ii) $\rightarrow$ (iii) let $u(\cdot)$ be an element of $C^{n}\left(\mathbf{R}^{+},[D(A)]\right)$. From the closedness of $B$ and $D(A) \subset D(B)$ we conclude that $B$ is a continuous operator from $[D(A)]$ into $E$. Looking at

$$
\mathbf{R}^{+} \stackrel{u(\cdot) \in C^{n}}{\rightarrow}[D(A)] \stackrel{B}{\rightarrow} E
$$

we see that $B u(\cdot) \in C^{n}\left(\mathbf{R}^{+}, E\right)$. The implication (iii) $\rightarrow(\mathrm{i})$ is obvious.

Now let $\left(x_{0}, x_{1}, \ldots, x_{n}\right) \in D(A)^{n+1}$ and define

$$
\begin{aligned}
\left(y_{0}, y_{1}, \ldots, y_{n}\right) & :=\Psi\left(x_{0}, x_{1}, \ldots, x_{n}\right) \\
& =\left(-\sum_{i+j=k-1} B_{i} x_{j}+x_{k}\right)_{0 \leq k \leq n} \in D\left(\Delta^{n+1}\right) .
\end{aligned}
$$

Using Theorems 2 and 3 we see that there exists a unique solution $u(\cdot): \mathbf{R}^{+} \rightarrow D(\Delta)$ in $C^{n+1}\left(\mathbf{R}^{+}, E^{n+1}\right)$ of

$$
u^{\prime}(t)=\Delta u(t), \quad u(0)=\left(y_{0}, y_{1}, \ldots, y_{n}\right) .
$$


This implies that there are functions $u_{i}(\cdot) \in C^{n+1}\left(\mathbf{R}^{+}, E\right), 1 \leq i \leq n+1$, with $u_{1}(\cdot): \mathbf{R}^{+} \rightarrow D(A),\left(u_{1}(0), u_{2}(0), \ldots, u_{n+1}(0)\right)=\left(y_{0}, y_{1}, \ldots, y_{n}\right)$ and

$$
\begin{aligned}
A u_{1}(t)+u_{2}(t) & =u_{1}^{\prime}(t) \\
B_{1} u_{1}(t)+u_{3}(t) & =u_{2}^{\prime}(t)
\end{aligned}
$$

(1)

$$
\begin{aligned}
B_{n-1} u_{1}(t)+u_{n+1}(t) & =u_{n}^{\prime}(t) \\
B_{n} u_{1}(t) & =u_{n+1}^{\prime}(t) .
\end{aligned}
$$

By the closedness of $A, B_{1}, \ldots, B_{n}$ and the differentiability of the functions $u_{i}(\cdot)$ it is easy to see that

$$
u_{1}^{(j)}(\cdot): \mathbf{R}^{+} \rightarrow D(A) \text { and } \frac{d}{d t} A u_{1}^{(j)}(\cdot)=A u_{1}^{(j+1)}(\cdot)
$$

for $0 \leq j \leq n-1$. Hence, by Lemma $5.2, u_{1}(\cdot)$ is an element of $C^{n+1}\left(\mathbf{R}^{+}, E\right) \cap$ $C^{n}\left(\mathbf{R}^{+},[D(A)]\right)$.

Now, looking at (1), we obtain

$$
u_{1}^{(n+1)}(t)-A u_{1}^{(n)}(t)-\sum_{k=1}^{n} B_{k} u_{1}^{(n-k)}(t)=0
$$

and

$$
u_{1}^{(k)}(0)=A u_{1}^{(k-1)}(0)+\sum_{j=1}^{k-1} B_{j} u_{1}^{(k-1-j)}(0)+u_{k+1}(0)
$$

for $1 \leq k \leq n$. Therefore

$$
\begin{gathered}
u_{1}^{(0)}(0)=u_{1}(0)=y_{0}=x_{0}, \\
u_{1}^{(1)}(0)=A u_{1}(0)+u_{2}(0)=A x_{0}+y_{1}=x_{1}, \\
u_{1}^{(2)}(0)=A x_{1}+B_{1} x_{0}+y_{2}=\sum_{i+j=1} B_{i} x_{j}+y_{2}=x_{2}
\end{gathered}
$$

and by induction we obtain

$$
\left(u_{1}^{(0)}(0), u_{1}^{(1)}(0), \ldots, u_{1}^{(n)}(0)\right)=\left(x_{0}, x_{1}, \ldots, x_{n}\right) .
$$

We have shown: given $\bar{x}=\left(x_{0}, x_{1}, \ldots, x_{n}\right) \in D(A)^{n+1}$, then the first coordinate $u_{1}(\cdot)$ of the solution of

$$
u^{\prime}(t)=\Delta u(t), \quad u(0)=\Psi \bar{x}
$$

is a solution of $\left(\mathrm{ACP}_{n+1}\right)$ in $C^{n+1}\left(\mathbf{R}^{+}, E\right) \cap C^{n}\left(\mathbf{R}^{+},[D(A)]\right)$ with initial value $\left(x_{0}, x_{1}, \ldots, x_{n}\right)$.

Suppose there is another solution $v(\cdot)$ of $\left(\mathrm{ACP}_{n+1}\right)$ in

$$
C^{n+1}\left(\mathbf{R}^{+}, E\right) \cap C^{n}\left(\mathbf{R}^{+},[D(A)]\right)
$$

with initial value $\left(x_{0}, \ldots, x_{n}\right)$. Then $w(\cdot):=v(\cdot)-u_{1}(\cdot)$ is a solution of $\left(\mathrm{ACP}_{n+1}\right)$ in $C^{n+1}\left(\mathbf{R}^{+}, E\right) \cap C^{n}\left(\mathbf{R}^{+},[D(A)]\right)$ with

$$
w^{(k)}(0)=0 \quad \text { for } 0 \leq k \leq n .
$$


Define $z(t):=\left(w_{1}(t), \ldots, w_{n+1}(t)\right)$ where $w_{1}(t):=w(t)$ and

$$
w_{k}(t):=w^{(k-1)}(t)-A w^{(k-2)}(t)-\sum_{j=1}^{k-2} B_{j} w^{(k-2-j)}(t) .
$$

The function $z(\cdot): \mathbf{R}^{+} \rightarrow D(\Delta)=D(A) \times E^{n}$ is an element of $C^{1}\left(\mathbf{R}^{+}, E^{n+1}\right.$ ) (see Lemma $5.2($ iii) ) with $z(0)=(0,0, \ldots, 0)$.

It is easy to see that

$$
w_{k}^{\prime}(t)=w_{k+1}(t)+B_{k-1} w(t), \quad 1 \leq k \leq n .
$$

Hence

$$
\begin{aligned}
\Delta z(t)= & \left(A w_{1}(t)+w_{1}^{\prime}(t)-A w_{1}(t), B_{1} w_{1}(t)+w_{3}(t), \ldots\right. \\
& \left.B_{n-1} w_{1}(t)+w_{n-1}(t), B_{n} w_{1}(t)\right) \\
= & \left(w_{1}^{\prime}(t), w_{2}^{\prime}(t), \ldots, w_{n}^{\prime}(t), B_{n} w_{1}(t)\right)
\end{aligned}
$$

and

$$
w_{n+1}^{\prime}(t)=w^{(n+1)}(t)-A w^{(n)}(t)-\sum_{k=1}^{n-1} B_{k} w^{(n-k)}(t)=B_{n} w_{1}(t)
$$

(since $w_{1}(\cdot)$ is a solution of $\left.\left(\mathrm{ACP}_{n+1}\right)\right)$. Therefore $\Delta z(t)=z^{\prime}(t), z(0)=0$. But $\Delta$ is a semigroup generator and therefore $z(\cdot)=0, w(\cdot)=0$ or $u_{1}(\cdot)=v(\cdot)$.

(b) The equivalence of statements (ii) and (iii) follows from the following considerations. Let $u(\cdot)$ be a solution of $\left(\mathrm{ACP}_{n+1}\right)$ in $C^{n+1}\left(\mathbf{R}^{+}, E\right) \cap C^{n}\left(\mathbf{R}^{+},[D(A)]\right)$. Then $u^{(n-k)}(\cdot): \mathbf{R}^{+} \rightarrow D(A) \subset D\left(B_{k}\right)$ for $0 \leq k \leq n\left(B_{0}:=A\right)$ and by Lemma 5.2 we obtain the continuity of $B_{k} u^{(n-k)}(\cdot)$ for $1 \leq k \leq n$.

If $u(\cdot)$ is a solution of $\left(\mathrm{ACP}_{n+1}\right)$ with the properties (a) and (b) of statement (iii), then

$$
t \rightarrow A u^{(n)}(t)=u^{(n+1)}(t)-\sum_{k=1}^{n} B_{k} u^{(n-k)}(t)
$$

is a continuous function. Therefore

$$
u^{(n-1)}(t)=\int_{0}^{t} u^{(n)}(s) d s \in D(A) \quad \text { for every } t \geq 0
$$

and

$$
t \rightarrow A u^{(n-1)}(t)=u^{(n)}(t)-\int_{0}^{t} \sum_{k=1}^{n} B_{k} u^{(n-k)}(s) d s
$$

is continuously differentiable. By induction we obtain

(1) $u^{(j)}(t) \in D(A)$ for every $t \geq 0$ and every $0 \leq j \leq n$,

(2) $A u^{(j)}(\cdot)$ are continuously differentiable for $0 \leq j \leq n-1$.

Hence $u(\cdot) \in C^{n+1}\left(\mathbf{R}^{+}, E\right), u(\cdot): \mathbf{R}^{+} \rightarrow D(A)$ and $A u(\cdot) \in C^{n}\left(R^{+}, E\right)$. Therefore, by Lemma $5.2, u(\cdot) \in C^{n+1}\left(\mathbf{R}^{+}, E\right) \cap C^{n}\left(\mathbf{R}^{+},[D(A)]\right)$.

(c) (ii) $\rightarrow$ (i): Let $\left(y_{0}, y_{1}, \ldots, y_{n}\right) \in D\left(\Delta^{n+1}\right)$. Then, by Lemma 5.1 , there is a unique $\left(x_{0}, x_{1}, \ldots, x_{n}\right) \in D(A)^{n+1}$ with $\Psi\left(x_{0}, x_{1}, \ldots, x_{n}\right)=\left(y_{0}, y_{1}, \ldots, y_{n}\right)$ where $x_{0}:=y_{0}$ and $x_{k}:=\sum_{i+j=k-1} B_{i} x_{j}+y_{k}\left(B_{0}:=A\right)$. Let $u(\cdot) \in C^{n+1}\left(\mathbf{R}^{+}, E\right) \cap$ $C^{n}\left(\mathbf{R}^{+},[D(A)]\right)$ be the unique solution of $\left(\mathrm{ACP}_{n+1}\right)$ with initial value $\left(x_{0}, x_{1}, \ldots\right.$, $\left.x_{n}\right)$. We will show that for every $\left(y_{0}, y_{1}, \ldots, y_{n}\right) \in D\left(\Delta^{n+1}\right)$ the Cauchy problem 
$u^{\prime}(t)=\Delta u(t), u(0)=\left(y_{0}, y_{1}, \ldots, y_{n}\right)$ has a unique solution in $C^{n+1}\left(\mathbf{R}^{+}, E^{n+1}\right)$. Then, by Theorems 2 and $3, \Delta$ and hence $A$ is a semigroup generator.

Define $u_{0}(t):=u(t)$,

$$
u_{k}(t):=\sum_{j=k}^{n}\left[\int_{0}^{t} \frac{1}{(j-k) !}(t-s)^{j-k} B_{j} u_{0}(s) d s+\frac{1}{(j-k) !} t^{j-k} y_{j}\right]
$$

and $w(t)=\left(u_{0}(t), u_{1}(t), \ldots, u_{n}(t)\right)$. Then $w(0)=\left(y_{0}, y_{1}, \ldots, y_{n}\right)$ and, by Lemma $5.2, w(\cdot) \in C^{n+1}\left(\mathbf{R}^{+}, E^{n+1}\right)$. From the definition of the functions $u_{k}(\cdot)$ it follows that

$$
\begin{aligned}
B_{1} u_{0}(t)+u_{2}(t) & =u_{1}^{\prime}(t) \\
B_{2} u_{0}(t)+u_{3}(t) & =u_{2}^{\prime}(t) \\
& \vdots \\
B_{n-1} u_{0}(t)+u_{n}(t) & =u_{n-1}^{\prime}(t) \\
B_{n} u_{0}(t) & =u_{n}^{\prime}(t) .
\end{aligned}
$$

Therefore, in order to show that $w(\cdot)$ is a solution of the differential equation $u^{\prime}(t)=\Delta u(t)$, it suffices to prove the validity of the equation

$$
A u_{0}(t)+u_{1}(t)=u_{0}^{\prime}(t)
$$

Define

$$
\begin{aligned}
g(t) & :=A u_{0}(t)+\sum_{j=1}^{n} \int_{0}^{t} \frac{1}{(j-1) !}(t-s)^{j-1} B_{j} u_{0}(s) d s+\sum_{j=1}^{n} \frac{1}{(j-1) !} t^{j-1} y_{j} \\
& =A u_{0}(t)+u_{1}(t) .
\end{aligned}
$$

Then we obtain the equality $g(\cdot)=u_{0}^{\prime}(\cdot)$ from the equations

$$
\begin{aligned}
g(0) & =A x_{0}+y_{1}=x_{1}=u^{(1)}(0) \\
g^{\prime}(0) & =A x_{1}+B_{1} x_{0}+y_{2}=x_{2}=u^{(2)}(0) \\
& \vdots \\
g^{(n-1)}(0) & =\sum_{i+j=n-1} B_{i} x_{j}+y_{n}=x_{n}=u^{(n)}(0)
\end{aligned}
$$

and

$$
g^{(n)}(t)=A u_{0}^{(n)}(t)+\sum_{j=1}^{n} B_{k} u_{0}^{(n-j)}(t)=u^{(n+1)}(t) .
$$

Suppose $v(\cdot) \in C^{n+1}\left(\mathbf{R}^{+}, E^{n+1}\right)$ is a solution of

$$
u^{\prime}(t)=\Delta u(t), \quad u(0)=(0,0, \ldots, 0)
$$

By the same arguments as in the proof of the implication (i) $\rightarrow$ (ii) we obtain that the first coordinate of $v(\cdot)$, denoted by $v_{0}(\cdot)$, is a solution of $\left(\mathrm{ACP}_{n+1}\right)$ in $C^{n+1}\left(\mathbf{R}^{+}, E\right) \cap C^{n}\left(\mathbf{R}^{+},[D(A)]\right)$ with initial value $(0, \ldots, 0)$. Hence $v_{0}(\cdot)=0$ and 
from

$$
\begin{gathered}
A v_{0}(t)+v_{1}(t)=v_{0}^{\prime}(t) \\
B_{1} v_{0}(t)+v_{2}(t)=v_{1}^{\prime}(t) \\
\vdots \\
B_{n-1} v_{0}(t)+v_{n}(t)=v_{n-1}^{\prime}(t)
\end{gathered}
$$

we obtain $v(\cdot)=\left(v_{0}(\cdot), v_{1}(\cdot), \ldots, v_{n}(\cdot)\right)=0$. Therefore $w(\cdot) \in C^{n+1}\left(\mathbf{R}^{+}, E^{n+1}\right)$ is the unique solution of the Cauchy problem $u^{\prime}(t)=\Delta u(t), u(0)=\left(y_{0}, y_{1}, \ldots, y_{n}\right) \in$ $D\left(\Delta^{n+1}\right)$.

The most restrictive assumption in Theorem 5 is $D(A) \subset D\left(B_{k}\right)$ for $1 \leq k \leq n$. If $A$ is a bounded operator, then the statement of the theorem is well known (every $B_{k}$ has to be bounded then). For bounded $A$ in general nothing can be said about existence of solutions of $\left(\mathrm{ACP}_{n+1}\right)$ if one of the operators $B_{i}$ is unbounded.

For damped second order problems

$$
\begin{aligned}
& u^{\prime \prime}(t)-A u^{\prime}(t)-B u(t)=0, \\
& u(0)=x, \quad u^{\prime}(0)=y
\end{aligned}
$$

answers can be given if $B$ is a cosine family generator and $A$ is a bounded operator commuting with the resolvent of $B$. For a summary of cosine family theory and references to the literature we refer to [17 and 23]. In the next theorem we have to know a Banach space $F$ which appears in the following characterization of a cosine family generator, due to Kisyńsky [15]. A densely defined linear operator $B$ on a Banach space $E$ is the generator of a strongly continuous cosine family if and only if

(i) there is a Banach space $F$ with $D(B) \subset F \subset E$ such that the Banach space topology of $F$ is not weaker than the topology induced in $F$ by $E$.

(ii) The operator $\left[\begin{array}{ll}0 & I \\ B & 0\end{array}\right]$ with domain $D(B) \times F$ is the generator of a strongly continuous group on the Banach space $F \times E$.

The space $F$ is given as the closure of $D(B)$ under the norm

$$
\|x\|_{F}:=\|x\|+\sup \left\{\left\|\frac{1}{n !}(\lambda-w)^{n+1}\left(\frac{-d^{n}}{d \lambda^{n}}\right) \lambda R\left(\lambda^{2}, A\right) x\right\|, \lambda>w, n \in \mathbf{N}\right\}
$$

(see [30]). If $B$ is unbounded then $[D(B)] \neq F \neq E$. Fattorini [7], Sova [28] and Da Prato-Giusti [3] proved that well-posedness of the undamped second order Cauchy problem (corresponding to $A=0$ ) is equivalent to $B$ being the generator of a strongly continuous cosine family. For a unified treatment of their results see [21]. In the proof of the next theorem we make use of their result and of a perturbation theorem (see $[\mathbf{5}, \mathbf{1 3}]$ ) which says that if $B$ is a cosine family generator and $C$ is bounded, then $B+C$ is a cosine family generator too.

THEOREM 6. Let $B$ be a densely defined linear operator on a Banach space $E$. Consider the following damped second order abstract Cauchy problem:

$$
\begin{aligned}
& u^{\prime \prime}(t)-A u^{\prime}(t)-B u(t)=0, \\
& u(0)=x, \quad u^{\prime}(0)=y .
\end{aligned}
$$

Then the following statements are equivalent:

(i) $B$ is the generator of a strongly continuous cosine family. 
(ii) $\rho(B) \neq \varnothing$ and, for every $x \in D(B), y \in F$ and every bounded operator $A$ with $A R(\lambda, B)=R(\lambda, B) A$, (\#) has a unique solution $u(\cdot): \mathbf{R} \rightarrow D(B)$ in $C^{2}(\mathbf{R}, E)$. For every sequence of solutions of (\#) with

$$
\lim _{n \rightarrow \infty} u_{n}(0)=\lim _{n \rightarrow \infty} u_{n}^{(1)}(0)=0
$$

we have $u_{n}(\cdot) \rightarrow 0$ uniformly on compacts of $\mathbf{R}$.

ProOF. (ii) $\Rightarrow$ (i) follows from the fact that the undamped equation is wellposed if and only if $B$ is a cosine family generator. To prove (i) $\Rightarrow$ (ii) let $B$ be a cosine family generator and $A$ a bounded operator with $A R(\lambda, B)=R(\lambda, B) A$. Then $B+\frac{1}{4} A^{2}$ is a cosine family generator, $A: D(B) \rightarrow D(B)$ and the second order Cauchy problem

$$
\begin{aligned}
& u^{(2)}(t)-\left(B+\frac{1}{4} A^{2}\right) u(t)=0, \\
& u(0)=x, \quad u^{(1)}(0)=y-\frac{1}{2} A x
\end{aligned}
$$

has a unique solution $u(\cdot): \mathbf{R} \rightarrow D(B)$ in $C^{2}(\mathbf{R}, E)$ for every $x \in D(B), y \in F$ (see, for example, [21]).

Define $v(t):=T(t) u(t)$ where $(T(t))$ is the group generated by $\frac{1}{2} A$. From $A R(\lambda, B)=R(\lambda, B) A$ we conclude that $T(t) R(\lambda, B)=R(\lambda, B) T(t), T(t): D(B) \rightarrow$ $D(B)$ and $B T(t)=T(t) B$ on $D(B)$. Moreover,

or

$$
\begin{aligned}
& v^{(1)}(t)=\frac{1}{2} A T(t) u(t)+T(t) u^{(1)}(t), \\
& v^{(2)}(t)=\frac{1}{2} A^{2} v(t)+A T(t) u^{(1)}(t)+B v(t),
\end{aligned}
$$

$$
\begin{aligned}
& v^{(2)}(t)-A v^{(1)}(t)-B v(t)=0, \\
& v(0)=x, \quad v^{(1)}(0)=y .
\end{aligned}
$$

Suppose there is another solution $g(\cdot)$ of $(\#)$ with $g(\cdot): \mathbf{R} \rightarrow D(B)$ and $g(\cdot) \in$ $C^{2}(\mathbf{R}, E)$. Then $h(\cdot):=g(\cdot)-v(\cdot)$ is a solution of

$$
\begin{aligned}
& u^{(2)}(t)-A u^{(1)}(t)-B u(t)=0, \\
& u(0)=0, \quad u^{(1)}(0)=0
\end{aligned}
$$

and $f(\cdot):=T(-\cdot) h(\cdot)$ a solution of

$$
\begin{aligned}
& u^{(2)}(t)-\left(B+\frac{1}{4} A^{2}\right) u(t)=0, \\
& u(0)=0, \quad u^{(1)}(0)=0 .
\end{aligned}
$$

Therefore $f(\cdot)=0$ and, by the bijectivity of $T(-t), h(\cdot)=0$ or $g(\cdot)=v(\cdot)$.

Let $h_{n}(\cdot)$ be a sequence of solutions of (\#) such that

$$
\lim _{n \rightarrow \infty} h_{n}(0)=\lim _{n \rightarrow \infty} h_{n}^{(1)}(0)=0 .
$$

Define $f_{n}(\cdot):=T(-\cdot) h_{n}(\cdot)$. Then the functions $f_{n}(\cdot)$ are solutions of

$$
\begin{aligned}
& u^{(2)}(t)-\left(B+\frac{1}{4} A^{2}\right) u(t)=0, \\
& u(0)=h_{n}(0), \quad u^{(1)}(0)=h_{n}^{(1)}(0)-\frac{1}{2} A h_{n}(0) .
\end{aligned}
$$

But then $f_{n}(\cdot)$ and hence $h_{n}(\cdot)$ converge to zero uniformly on compacts of $\mathbf{R}$.

Another important case of

$$
u^{\prime \prime}(t)-A u^{\prime}(t)-B u(t)=0
$$


is the class of equations in which $A$ and $B$ are densely defined operators with $D(B) \subset D(A)$. Sandefur [25-27] and Aviles and Sandefur [1] discussed iterated differential equations

(\#\#)

$$
\prod_{i=1}^{n}\left(\frac{d}{d t}-A_{i}\right) w(t)=0
$$

where $A_{i}$ are semigroup generators on a Banach space $E$ such that

$$
D:=\left\{D\left(A_{i(1)} \cdots A_{i(m)}\right) ; i(j) \in\{1, \ldots, n\}, m=1,2, \ldots\right\}
$$

is dense in $E$. For $n=2$ (\#\#) becomes

(\#\#\#)

$$
w^{(2)}(t)-\left(A_{1}+A_{2}\right) w^{(1)}(t)-A_{1} A_{2} w(t)=0 .
$$

Sandefur proved well-posedness of (\#\#) for every $1 \leq k \leq n$ (along the lines of Fattorini) under the assumptions on the operators $A_{i}$ mentioned above. Therefore, in the case that $D(B)$ is contained in $D(A)$ one obtains well-posedness if the operator $A$ is the sum and the operator $B$ the product of two semigroup generators.

For further results on higher order Cauchy problems and references to the literature we refer to $[\mathbf{7}, \mathbf{1 0}, \mathbf{1 1}, \mathbf{1 3}, \mathbf{1 8}, \mathbf{3 1}]$ and a paper of Weiss $[\mathbf{3 2}]$ which was, together with [26], the starting point of our investigations.

4. Explicit solutions of higher order Cauchy problems. In this section we show how to get an explicit expression for the solution of $\left(\mathrm{ACP}_{n+1}\right)$. As proved in the previous section the solution of $\left(\mathrm{ACP}_{n+1}\right)$ is given by the first coordinate of the semigroup $\Gamma(t)$ generated by $\Delta$ as defined in Theorem 3 . The first and main step to obtain this "first coordinate" is the formula for $R(\lambda, \Delta)$ given in the next proposition.

Proposition 7. Let $A, B_{1}, B_{2}, \ldots, B_{n}$ be densely defined closed operators and let $D(A) \subset D\left(B_{k}\right)$ for $1 \leq k \leq n$. Define $\Delta$ as in Theorem 3. Then the following statements are equivalent:

(i) $\lambda \in \rho(\Delta)$.

(ii) $R_{\lambda}:=\left(\lambda^{n}(\lambda-A)-\lambda^{n-1} B_{1}-\cdots-B_{n}\right)^{-1}$ exists.

(Remark: in the scalar case $R_{\lambda}$ corresponds to $\operatorname{det}(\lambda-\Delta)^{-1}$.) If $\lambda \in \rho(\Delta)$ then $R(\lambda, \Delta)$ is given by

$$
\left[\begin{array}{cccccc}
\lambda^{n} R_{\lambda} & \lambda^{n-1} R_{\lambda} & \lambda^{n-2} R_{\lambda} & \cdot & \cdot & R_{\lambda} \\
Y_{1} R_{\lambda} & \lambda^{n-1} X_{1} R_{\lambda} & \lambda^{n-2} X_{1} R_{\lambda} & \cdot & \cdot & X_{1} R_{\lambda} \\
\lambda Y_{2} R_{\lambda} & Y_{2} R_{\lambda} & \lambda^{n-2} X_{2} R_{\lambda} & \cdot & \cdot & X_{2} R_{\lambda} \\
\cdot & \cdot & \cdot & \cdot & \cdot & \cdot \\
\cdot & \cdot & \cdot & \cdot & \cdot & \cdot \\
\lambda^{n-1} Y_{n} R_{\lambda} & \lambda^{n-2} Y_{n} R_{\lambda} & \cdot & Y_{n} R_{\lambda} & \cdot & X_{n} R_{\lambda}
\end{array}\right]
$$

where

$$
\begin{aligned}
X_{1} & :=\lambda-A, \\
X_{k} & :=\left(\lambda^{k-1}(\lambda-A)-\lambda^{k-2} B_{1}-\cdots-B_{k-1} \quad(k \geq 2)\right. \\
Y_{k}: & =\sum_{j=k}^{n} \lambda^{n-k} B_{k} .
\end{aligned}
$$


ProOF. (i) $\rightarrow$ (ii). Let $\lambda \in \rho(\Delta)$. Then there are bounded operators $X_{i j}$ on $E$ such that

$$
\left(X_{i j}\right)(\lambda-\Delta)=(\lambda-\Delta)\left(X_{i j}\right)=I .
$$

This leads to the following equations:

$$
\begin{aligned}
& X_{1, n+1}\left(\lambda^{n}(\lambda-A)-\lambda^{n-1} B_{1}-\cdots-B_{n}\right)=I \\
& X_{1, k}=\lambda^{n+1-k} X_{1, n+1} \quad \text { for } 1 \leq k \leq n .
\end{aligned}
$$

$$
\begin{aligned}
& X_{i, 1}(\lambda-A)-X_{i, 2} B_{1}-\cdots-X_{i, n+1} B_{n}=0 \quad(2 \leq i \leq n+1), \\
& X_{i, k}=\lambda^{n+1-k} X_{i, n+1} \quad \text { for } i \leq k \leq n+1,
\end{aligned}
$$

and $X_{i, k}=\lambda^{i-1-k}\left(\lambda^{n+2-i} X_{i, n+1}-I\right)$ for $k \leq i-1$.

$$
(\lambda-A) X_{1,1}-X_{2,1}=I
$$

and $(\lambda-A) X_{1, k}-X_{2, k}=0$ for $2 \leq k \leq n+1$.

$$
-B_{i} X_{1, k}+\lambda X_{i+1, k}-X_{i+2, k}=\delta_{i+1, k} I
$$

for $1 \leq i \leq n-1$ and $1 \leq k \leq n+1$.

$$
-B_{n} X_{1, k}+\lambda X_{n+1, k}=\delta_{n+1, k} I \text { for } 1 \leq k \leq n+1 .
$$

Looking at (3) and (4) we obtain:

(6)

$$
\begin{aligned}
(\lambda-A) X_{1, n+1} & =X_{2, n+1} \\
-B_{1} X_{1, n+1}+\lambda X_{2, n+1} & =X_{3, n+1} \\
-B_{2} X_{1, n+1}+\lambda X_{3, n+1} & =X_{4, n+1}
\end{aligned}
$$

$$
\begin{aligned}
& -B_{n-1} X_{1, n+1}+\lambda X_{n, n+1}=X_{n+1, n+1} \\
& -B_{n} X_{1, n+1}+\lambda X_{n+1, n+1}=I .
\end{aligned}
$$

From (6) we obtain

$$
\left(\lambda^{n}(\lambda-A)-\lambda^{n-1} B_{1}-\cdots-B_{n}\right) X_{1, n+1}=I .
$$

Therefore, by (1) and (7),

$$
R_{\lambda}:=X_{1, n+1}=\left(\lambda^{n}(\lambda-A)-\lambda^{n-1} B_{1}-\cdots-B_{n}\right)^{-1}
$$

exists. By (6) we obtain expressions for $X_{k, n+1}$ for $2 \leq k \leq n+1$ and by (1)-(3) for all $X_{i, k}$. This leads to the formula for $R(\lambda, \Delta)$ stated in the proposition.

(ii) $\rightarrow$ (i). If $R_{\lambda}$ exists, then define $R(\lambda, \Delta)$ as above and show that

$$
R(\lambda, \Delta)(\lambda-\Delta)=(\lambda-\Delta) R(\lambda, \Delta)=I .
$$

We remark that no commutativity of the operators $R_{\lambda}, A, B_{1}, \ldots, B_{n}$ is needed to prove the first equality. 
COROLLARY 8. Let $A$ be the generator of a strongly continuous semigroup and let $B_{1}, B_{2}, \ldots, B_{n}$ be closed operators with $D(A) \subset D\left(B_{k}\right)$ for $1 \leq k \leq n$. Then there exists a real number $w$ such that, for every $\lambda \in \mathbf{C}$ with $\operatorname{Re} \lambda>w, R_{\lambda}:=$ $\left(\lambda^{n}(\lambda-A)-\lambda^{n-1} B_{1}-\cdots-B_{n}\right)^{-1}$ exists. Let $p>w$ and define $\gamma$ as the path $\{p+i \mathbf{R}\}$. Then a solution of $\left(\mathrm{ACP}_{n+1}\right)$ is given by

$$
\frac{1}{2 \pi i} \int_{\gamma} e^{\lambda t} \sum_{k=0}^{n} \lambda^{n-k} R_{\lambda}\left[\left(-\sum_{j=0}^{k-1} B_{k-1-j} x_{j}\right)+x_{k}\right] d \lambda
$$

for every initial value $\left(x_{0}, x_{1}, \ldots, x_{n}\right) \in(D(A))^{n+1}$.

ProOF. Let $\bar{x}:=\left(x_{0}, x_{1}, \ldots, x_{n}\right) \in(D(A))^{n+1}$. Define $\Psi$ as in the proof of Theorem 5(a). Then

$$
\Psi\left(x_{0}, x_{1}, \ldots, x_{n}\right)=\left(z_{0}, z_{1}, \ldots, z_{n}\right)
$$

where

$$
z_{k}=-\sum_{j=0}^{k-1} B_{k-1-j} x_{j}+x_{k} \quad\left(B_{0}:=A\right) .
$$

Define $\Delta$ as in Theorem 3. Then, as shown in the proof of Theorem 5 , a solution of $\left(\mathrm{ACP}_{n+1}\right)$ is given as the first coordinate of $\Gamma(t) \Psi \bar{x}$, where $\Gamma(t)$ is the semigroup generated by $\Delta$. Using the inversion formula for the Laplace transform

$$
\Gamma(t) \Psi \bar{x}=\frac{1}{2 \pi i} \int_{\gamma} e^{\lambda t} R(\lambda, \Delta) \Psi \bar{x} d \lambda
$$

(for a proof use $[\mathbf{3 3}$, p. 66]; or [4]) and Proposition 7 we obtain the expression for the solution of $\left(\mathrm{ACP}_{n+1}\right)$ stated in the corollary.

At first glance the formula for the solution as given in Corollary 8 seems to be rather difficult. However, there are a lot of examples in which an explicit expression for the solution can be easily determined by the formula above (see the examples at the end of the next section and at the end of the paper).

5. Well-posedness of higher order abstract Cauchy problems. The Cauchy problem $\left(\mathrm{ACP}_{n+1}\right)$ for

$$
u^{(n+1)}(t)-A u^{(n)}(t)-\sum_{k=1}^{n} B_{k} u^{(n-k)}(t)=0
$$

is called well-posed if

(i) $\left(\mathrm{ACP}_{n+1}\right)$ is solvable for a dense subset $D$ of initial data $\bar{x}=\left(x_{0}, x_{1}, \ldots, x_{n}\right)$ in $E^{n+1}$.

(ii) The solutions depend continuously on their initial data; i.e.: Let $\left(v_{k}(\cdot)\right)$ be a sequence of solutions of $\left(\mathrm{ACP}_{n+1}\right)$ such that $v_{k}^{(j)}(0) \rightarrow 0$ in $E$ for $k \rightarrow \infty$ and $0 \leq j \leq n$. Then $v_{k}(\cdot) \rightarrow 0$ uniformly on compacts of $R^{+}$.

We remark that assumption (ii) implies uniqueness of the solutions and we start our discussion of well-posedness with a fundamental lemma which will be of frequent use in the following (for a version of the lemma for $n=1$, see $\S 6$ ). 
LEMMA 9. Let $A$ be the generator of a strongly continuous semigroup and let $B_{1}, B_{2}, \ldots, B_{n}$ be closed operators with $D(A) \subset D\left(B_{k}\right)$ for $1 \leq k \leq n$. Then there are strongly continuous families $\left(T_{i, j}(t)\right)(t \geq 0,0 \leq i, j \leq n)$ of bounded linear operators such that the semigroup $\Gamma(t)$ generated by

$$
\Delta:=\left[\begin{array}{cccccc}
A & I & 0 & . & . & 0 \\
B_{1} & 0 & I & . & . & 0 \\
B_{2} & 0 & 0 & . & . & 0 \\
\cdot & . & . & . & . & . \\
. & . & . & . & . & I \\
B_{n} & 0 & 0 & . & . & 0
\end{array}\right]
$$

is given by

$$
\left[\begin{array}{cccc}
T_{0,0}(t) & \cdot & \cdot & T_{0, n}(t) \\
\cdot & \cdot & \cdot & \cdot \\
\cdot & \cdot & \cdot & \cdot \\
\cdot & \cdot & \cdot & \cdot \\
T_{n, 0}(t) & \cdot & \cdot & T_{n, n}(t)
\end{array}\right] \cdot
$$

The families $\left(T_{i, j}(t)\right)$ have the following properties:

(a) $T_{0,0}(t): D(A) \rightarrow D(A)$ and $T_{0, j}(t): E \rightarrow D(A)$ for $1 \leq j \leq n$.

(b) If $0 \leq i \leq n-1$ and $1 \leq j \leq n$, then $t \rightarrow T_{i, j}(t) x$ is continuously differentiable for every $x \in E$ and (where necessary, define $B_{0}:=A$ )

$$
T_{i, j}^{\prime}(t) x=T_{i, j-1}(t) x=B_{i} T_{0, j}(t) x+T_{i+1, j}(t) x .
$$

(c) If $0 \leq i \leq n-1$ and $j=0$, then $t \rightarrow T_{i, 0}(t) x$ is continuously differentiable for every $x \in D(A)$ and

$$
T_{i, 0}^{\prime}(t) x=\sum_{k=0}^{n} T_{i, k}(t) B_{k} x=B_{i} T_{0,0}(t)+T_{i+1,0}(t) x .
$$

(d) If $i=n$ and $j=0$, then $t \rightarrow T_{n, 0}(t) x$ is continuously differentiable for every $x \in D(A)$ and

$$
T_{n, 0}^{\prime}(t) x=\sum_{k=0}^{n} T_{n, k}(t) B_{k} x=B_{n} T_{0,0}(t) x .
$$

(e) If $i=n$ and $1 \leq j \leq n$, then $t \rightarrow T_{n, j}(t) x$ is continuously differentiable for every $x \in E$ and

$$
T_{n, j}^{\prime}(t) x=T_{n, j-1}(t) x=B_{n} T_{0, j}(t) x .
$$

(f) Let $\bar{x}:=\left(x_{0}, x_{1}, \ldots, x_{n}\right) \in(D(A))^{n+1}$. Then

$$
\begin{aligned}
v(t) & :=\sum_{k=0}^{n} T_{0, k}(t)\left[x_{k}-\sum_{j=0}^{k-1} B_{k-1-j} x_{j}\right] \\
& =\sum_{i=0}^{n-1}\left[T_{0, i}(t) x_{i}-\sum_{k=i+1}^{n} T_{0, k}(t) B_{k-1-i} x_{i}\right]+T_{0, n}(t) x_{n}
\end{aligned}
$$

is a solution of $\left(\mathrm{ACP}_{n+1}\right)$ with initial value $\bar{x}$. 
ProOF. Statements (a)-(e) follow from the facts that $D(\Delta)$ is given by $D(A) \otimes E^{n}, \Gamma(t): D(\Delta) \rightarrow D(\Delta)$ and that $(d / d t) \Gamma(t) \bar{x}=\Delta \Gamma(t) \bar{x}=\Gamma(t) \Delta \bar{x}$ for every $\bar{x} \in D(\Delta)$. Statement (f) follows from the proof of Theorem 5 .

COROLLARY 10. Let $A$ be the generator of a strongly continuous semigroup and let $B_{1}, B_{2}, \ldots, B_{n}$ be closed linear operators with $D(A) \subset D\left(B_{k}\right)$ for $1 \leq k \leq n$. Let $u_{k}(\cdot)$ be a sequence of solutions of $\left(\mathrm{ACP}_{n+1}\right)$ in $C^{n+1}\left(\mathbf{R}^{+}, E\right) \cap C^{n}\left(\mathbf{R}^{+},[D(A)]\right)$ with $u_{k}^{(j)}(0) \rightarrow 0$ in $[D(A)]$ for $0 \leq j \leq n-1, u_{k}^{(n)}(0) \in D(A)$ for all $k$ and $u_{k}^{(n)}(0) \rightarrow 0$ in $E$. Then $u_{k}(\cdot) \rightarrow 0$ in $E$ uniformly on compacts of $\mathbf{R}^{+}$.

Proof. The initial values are all in $D(A)$. Therefore, by Theorem 5 and the previous lemma, the solutions are unique and given by

$$
u(t)=\sum_{k=0}^{n} T_{0, k}(t)\left[u^{(k)}(0)-\sum_{j=0}^{k-1} B_{k-1-j} u^{(j)}(0)\right] .
$$

The statement of the corollary follows now from $\left\|T_{i, j}(t)\right\| \leq\|\Gamma(t)\| \leq M e^{w t}$ and the continuity of the operators $B_{k}$ from the Banach space $[D(A)]$ into $E$.

In order to motivate the hypothesis of the following theorem we will consider for a moment the second order equation

$$
u^{(2)}(t)-A u^{(1)}(t)-B u(t)=0 .
$$

Examples are the strongly damped wave equation

$$
u^{(2)}(t)-a \Delta u^{(1)}(t)-\Delta u(t)=0, \quad a>0,
$$

the linear strongly damped Klein-Gordon equation

$$
u^{(2)}(t)-2 a \Delta u^{(1)}(t)-(\Delta+b I) u(t)=0
$$

and the vibrating beam equation

$$
u^{(2)}(t)+2\left(a \Delta^{2}+b\right) u^{(1)}(t)+\Delta^{2} u(t)=0 .
$$

We see that (\#) frequently appears in the form

$$
u^{(2)}(t)-(a A+b I) u^{(1)}(t)-(c A+d I) u(t)=0, \quad a>0, b, c, d \in \mathbf{C} .
$$

For a discussion of this equation see the example following Corollary 21. In this case

$$
R_{\lambda}=\frac{1}{a \lambda+c} R\left(\frac{1}{a \lambda+c}\left(\lambda^{2}-\lambda b-d\right), A\right)
$$

and hence, for $B:=c A+d I$ we obtain $B R_{\lambda}=R_{\lambda} B$ on $D(A)$, which is the additional assumption in the following theorem.

THEOREM 11. Let $A$ be the generator of a strongly continuous semigroup and let $B_{1}, B_{2}, \ldots, B_{n}$ be closed operators with

(i) $D(A) \subset D\left(B_{k}\right)$ for $1 \leq k \leq n$.

(ii) $B_{k} R_{\lambda}=R_{\lambda} B_{k}$ on $D(A)$ for $1 \leq k \leq n$ and $\lambda \in\{p+i \mathbf{R}\}, p>w$ ( $w$ defined as in Corollary 8). 
Then $\left(\mathrm{ACP}_{n+1}\right)$ is well-posed and every solution with initial value $\left(x_{0}, x_{1}\right.$, $\left.\ldots, x_{n}\right)$ is given by $\sum_{k=0}^{n} T_{n-k, n}(t) x_{k}$ where the operators $T_{n-k, n}(t)$ are defined as in Lemma 9.

Proof. (a) From $B_{k} R_{\lambda}=R_{\lambda} B_{k}$ on $D(A)$ for $1 \leq k \leq n$ it follows that $A R_{\lambda}=$ $R_{\lambda} A$ on $D(A)$. Let $\lambda, \mu \in\{p+i \mathbf{R}\}$. For every $x \in E$ define $y:=R_{\lambda} x \in D(A)$. Then

$$
\begin{aligned}
R_{\lambda} R_{\mu} x & =R_{\lambda} R_{\mu}\left(\lambda^{n}(\lambda-A)-\lambda^{n-1} B_{1}-\cdots-B_{n}\right) y \\
& =R_{\lambda}\left(\lambda^{n}(\lambda-A)-\lambda^{n-1} B_{1}-\cdots-B_{n}\right) R_{\mu} y=R_{\mu} R_{\lambda} x .
\end{aligned}
$$

Therefore $R_{\lambda} R_{\mu} x=R_{\mu} R_{\lambda} x$ for $\lambda, \mu \in\{p+i \mathbf{R}\}$.

(b) As seen in $\S 4$, the operators $T_{0, j}(t)(0 \leq j \leq n)$ are given as inverse Laplace transforms of the functions $\lambda \rightarrow \lambda^{n-j} R_{\lambda}$; i.e.,

$$
T_{0, j}(t) x=\frac{1}{2 \pi i} \int_{\gamma} e^{\lambda t} \lambda^{n-j} R_{\lambda} x d \lambda
$$

for every $x \in E$ (if $1 \leq j \leq n$ ) or for every $x \in D(A)$ (if $j=0$ ). Therefore, by the closedness of the operators $B_{k}\left(0 \leq k \leq n, B_{0}:=A\right)$, we obtain $T_{0, j}(t) B_{k}=$ $B_{k} T_{0, j}(t)$ on $D(A)$ for $0 \leq j, k \leq n$.

(c) By Lemma 9(f), a solution of $\left(\mathrm{ACP}_{n+1}\right)$ with initial value $\left(x_{0}, x_{1}, \ldots, x_{n}\right) \in$ $(D(A))^{n+1}$ is given by

$$
\sum_{i=0}^{n-1} S_{i}(t) x_{i}+T_{0, n}(t) x_{n}
$$

where

$$
\begin{aligned}
S_{i}(t) x & :=T_{0, i}(t) x-\sum_{k=i+1}^{n} T_{0, k}(t) B_{k-1-i} x \\
& =T_{0, i}(t) x-\sum_{k=i+1}^{n} B_{k-1-i} T_{0, k}(t) x
\end{aligned}
$$

for $x \in D(A)$. By Lemma $9(\mathrm{~b})$, we obtain

$$
S_{i}(t) x:=T_{0, i}(t) x-\sum_{k=0}^{n-i-1} T_{k, k+i}(t) x+\sum_{k=0}^{n-i-1} T_{k+1, k+1+i}(t) x=T_{n-i, n}(t) x .
$$

(d) Let $u(\cdot)$ be a solution of $\left(\mathrm{ACP}_{n+1}\right)$ with $u^{(j)}(0)=0$ for $0 \leq j \leq n$. Define $v(\cdot):=R_{\lambda} u(\cdot)$. Then $v(\cdot)$ is a solution of $\left(\mathrm{ACP}_{n+1}\right)$ with $v^{(j)}(0)=0$ for $0 \leq j \leq n$ and $B_{k} v^{(n-k)}(\cdot)=B_{k} R_{\lambda} u^{(n-k)}(\cdot)$ are continuous functions. Hence, by Theorem 5(iii), $v(\cdot)=R_{\lambda} u(\cdot)=0$ or $u(\cdot)=0$. Therefore, the solution of $\left(\mathrm{ACP}_{n+1}\right)$ is uniquely determined by its initial data.

(e) Let $\bar{x}=\left(x_{0}, x_{1}, \ldots, x_{n}\right) \in(D(A))^{n+1}$. Then the unique solution of $\left(\mathrm{ACP}_{n+1}\right)$ with initial value $\bar{x}$ is given by $\sum_{i=0}^{n} T_{n-i, n}(t) x_{i}$.

Let $\left(v_{k}(\cdot)\right)$ be a sequence of solutions of $\left(\mathrm{ACP}_{n+1}\right)$ such that $v_{k}^{(j)}(0) \rightarrow 0$ for $k \rightarrow \infty$ and $0 \leq j \leq n$. Define $u_{k}(\cdot):=R_{\mu} v_{k}(\cdot)$. Then $u_{k}(\cdot)$ is a solution of $\left(\mathrm{ACP}_{n+1}\right)$ with $u_{k}^{(j)}(0) \in D(A)$ for $0 \leq j \leq n$. Therefore

$$
u_{k}(t)=R_{\mu} v_{k}(t)=\sum_{i=0}^{n} T_{n-i, n}(t) R_{\mu} v_{k}^{(i)}(0)
$$


As seen in $\S 4$, the operators $T_{k, n}(t)(0 \leq k \leq n)$ are given as inverse Laplace transforms of the functions $\lambda \rightarrow X_{k} R_{\lambda}$ where $X_{0}=I, X_{1}=(\lambda-A)$,

$$
X_{k}=\left(\lambda^{k-1}(\lambda-A)-\lambda^{k-2} B_{1}-\cdots-B_{k-1}\right) \quad(k \geq 2) \text {; }
$$

i.e.,

$$
T_{k, n}(t) x=\frac{1}{2 \pi i} \int_{\gamma} e^{\lambda t} X_{k} R_{\lambda} x d \lambda \quad \text { for every } x \in E .
$$

Now let $\mu \in \gamma=\{p+i \mathbf{R}\}$. Then, as proved in (a),

for every $x \in E$. Therefore

$$
\begin{aligned}
T_{k, n}(t) R_{\mu} x & =\frac{1}{2 \pi i} \int_{\gamma} e^{\lambda t} X_{k} R_{\lambda} R_{\mu} x d \lambda \\
& =R_{\mu} \frac{1}{2 \pi i} \int_{\gamma} e^{\lambda t} X_{k} R_{\lambda} x d \lambda \\
& =R_{\mu} T_{k, n}(t) x
\end{aligned}
$$

$$
u_{k}(t)=R_{\mu} v_{k}(t)=R_{\mu}\left(\sum_{i=0}^{n} T_{n-i, n}(t) v_{k}^{(i)}(0)\right)
$$

or

$$
v_{k}(t)=\sum_{i=0}^{n} T_{n-i, n}(t) v_{k}^{(i)}(0)
$$

Hence, by

$$
\left\|T_{n-i, n}(t)\right\| \leq\|\Gamma(t)\| \leq M e^{w t},
$$

we obtain that $v_{k}(\cdot) \rightarrow 0$ uniformly on compacts of $\mathbf{R}^{+}$.

EXAMPLE. Let $A$ be the generator of a strongly continuous semigroup $(T(t))$ on a Banach space. Consider the well-posed Cauchy problem

$$
\begin{aligned}
& x^{(3)}(t)-A x^{(2)}(t)+c x^{(1)}(t)-c A x(t)=0, \\
& x(0)=x_{0}, \quad x^{(1)}(0)=x_{1}, \quad x^{(2)}(0)=x_{2}, \quad x_{i} \in D(A)
\end{aligned}
$$

where $c$ is any nonzero complex number. In this case

$$
R_{\lambda}=\left(\lambda^{2}(\lambda-A)+c \lambda I-c A\right)^{-1}=\left(\lambda^{2}+c\right)^{-1} R(\lambda, A) .
$$

Define

$$
z_{k}:=-\sum_{j=0}^{k-1} B_{k-1-j} x_{j}+x_{k}
$$

Then $z_{0}=x_{0}, z_{1}=-A x_{0}+x_{1}, z_{2}=c x_{0}-A x_{1}+x_{2}$ and the unique solution $v(\cdot)$ is given by

$$
\begin{aligned}
& \frac{1}{2 \pi i} \int_{\gamma} e^{\lambda t}\left(\lambda^{2}+c\right)^{-1}\left(\lambda^{2} R_{\lambda} z_{0}+\lambda R_{\lambda} z_{1}+R_{\lambda} z_{2}\right) d \lambda \\
& \quad=\frac{1}{2 \pi i} \int_{\gamma} e^{\lambda t}\left(\lambda^{2}+c\right)^{-1}\left(\lambda x_{0}+x_{1}+R(\lambda, A)\left(c x_{0}+x_{2}\right)\right) d \lambda
\end{aligned}
$$

By

$$
\left(\lambda^{2}+c\right)^{-1}=\sum_{n=0}^{\infty}\left(-c \lambda^{-2}\right)^{n}
$$


(b)

$$
\begin{gathered}
\frac{1}{2 \pi i} \int_{\gamma} \lambda^{-(k+1)} e^{\lambda t} d \lambda=\frac{1}{k !} t^{k} \\
\frac{1}{2 \pi i} \int_{\gamma} \lambda^{-(n+1)} e^{\lambda t} R(\lambda, A) x d \lambda=\int_{0}^{t} \frac{1}{n !}(t-s)^{n} T(s) x d s
\end{gathered}
$$

and

$$
\begin{aligned}
\sum_{n=0}^{\infty}(-c)^{n} \frac{1}{(2 n) !} t^{2 n} & =\cosh \sqrt{-c} t \\
\sum_{n=0}^{\infty}(-c)^{n} \frac{1}{(2 n+1) !} t^{2 n-1} & =\frac{1}{\sqrt{-c}} \sinh \sqrt{-c} t
\end{aligned}
$$

we obtain that the solution $v(\cdot)$ can be written as:

$$
\begin{aligned}
& (\cosh \sqrt{-c} t) x_{0}+\frac{1}{\sqrt{-c}}(\sinh \sqrt{-c} t) x_{1} \\
& \quad+\frac{1}{\sqrt{-c}} \int_{0}^{t}(\sinh \sqrt{-c}(t-s)) T(s)\left(c x_{0}+x_{2}\right) d s .
\end{aligned}
$$

EXAMPLE. Let $A$ be the generator of a strongly continuous semigroup $(T(t))$ on a Banach space. Consider the well-posed Cauchy problem

$$
\begin{aligned}
& x^{(3)}(t)-A x^{(2)}(t)-a A x^{(1)}(t)-\frac{3}{4} a^{2} x^{(1)}(t)-\frac{1}{4} a^{2} A x(t)-\frac{1}{4} a^{3} x(t)=0, \\
& x(0)=x_{0}, \quad x^{(1)}(0)=x_{1}, \quad x^{(2)}(0)=x_{2}, \quad x_{0}, x_{1}, x_{2} \in D(A),
\end{aligned}
$$

where $a$ is any nonzero complex number. Then

$$
\begin{gathered}
R_{\lambda}=(\lambda+a / 2)^{-2} R(\lambda-a, A), \\
z_{0}=x_{0}, \quad z_{1}=-A x_{0}+x_{1}, \\
z_{2}=-A\left(a x_{0}+x_{1}\right)+x_{2}-\frac{3}{4} a^{2} x_{0}
\end{gathered}
$$

and the solution is given by

$$
\frac{1}{2 \pi i} \int_{\gamma} e^{\lambda t}\left(\lambda+\frac{a}{2}\right)^{-2}\left[\lambda^{2} R(\lambda-a, A) z_{0}+\lambda R(\lambda-a, A) z_{1}+R(\lambda-a, A) z_{2}\right] d \lambda
$$

With similar calculations as in the previous example we get as the unique solution of the Cauchy problem:

$$
e^{-a / 2 \cdot t}\left[\int_{0}^{t}(t-s) e^{3 a / 2 \cdot s} T(s)\left(\frac{1}{4} a^{2} x_{0}+a x_{1}+x_{2}\right) d s+x_{0}+t\left(\frac{a}{2} x_{0}+x_{1}\right)\right] .
$$

6. Damped second order Cauchy problems. In this section we investigate damped second order Cauchy problems

$$
u^{(2)}(t)-A u^{(1)}(t)-B u(t)=f(t, u(t)), \quad u(0)=x, \quad u^{(1)}(0)=y
$$

which we will call semilinear if $f(\cdot, \cdot) \neq 0$ and inhomogeneous if $f(\cdot, u(\cdot))$ is independent of $u(\cdot)$; i.e., $f(t, u(t))=g(t)$ for some function $g(\cdot): \mathbf{R}^{+} \rightarrow E$. We show how the problem above is related to the initial value problem

$$
u^{(1)}(t)=A u(t)+\int_{0}^{t}(B u(s)+f(s, u(s)) d s, \quad u(0)=x,
$$

which will be called the integrated Cauchy problem.

We start with a version of Lemma 9 for $n=1$. 
LEMMA 12. Let $A$ be the generator of a strongly continuous semigroup on $E$ and let $B$ be a closed linear operator on $E$ with $D(A) \subset D(B)$. Then there are families $\left(T_{i}(t)\right), 1 \leq i \leq 4, t \geq 0$, of bounded linear operators on $E$ such that the semigroup $\Gamma(t)$ generated by $\Delta:=\left[\begin{array}{ll}A & I \\ B & 0\end{array}\right]$ is given by

$$
\left[\begin{array}{ll}
T_{1}(t) & T_{2}(t) \\
T_{3}(t) & T_{4}(t)
\end{array}\right] .
$$

For every $1 \leq i \leq 4$ and every $x \in E$ the map $t \rightarrow T_{i}(t) x$ is continuous and

(i) $T_{1}(0)=I, T_{1}(t): D(A) \rightarrow D(A), t \rightarrow T_{1}(t) x$ is continuously differentiable for every $x \in D(A)$ and

$$
T_{1}^{\prime}(t) x=A T_{1}(t) x+T_{3}(t) x=T_{1}(t) A x+T_{2}(t) B x,
$$

(ii) $T_{2}(0)=0, T_{2}(t): E \rightarrow D(A), t \rightarrow T_{2}(t) x$ is continuously differentiable for every $x \in E$ and

$$
T_{2}^{\prime}(t) x=T_{1}(t) x=A T_{2}(t) x+T_{4}(t) x,
$$

(iii) $T_{3}(0)=0, t \rightarrow T_{3}(t) x$ is continuously differentiable for every $x \in D(A)$ and

$$
T_{3}^{\prime}(t) x=B T_{1}(t) x=T_{3}(t) A x+T_{4}(t) B x
$$

(iv) $T_{4}(0)=I, t \rightarrow T_{4}(t) x$ is continuously differentiable for every $x \in E$ and

$$
T_{4}^{\prime}(t) x=B T_{2}(t) x=T_{3}(t) x
$$

(v)

$$
\begin{aligned}
& T_{1}(t+s)=T_{1}(s) T_{1}(t)+T_{2}(s) T_{3}(t), \\
& T_{2}(t+s)=T_{1}(s) T_{2}(t)+T_{2}(s) T_{4}(t), \\
& T_{3}(t+s)=T_{3}(s) T_{1}(t)+T_{4}(s) T_{3}(t), \\
& T_{4}(t+s)=T_{3}(s) T_{2}(t)+T_{4}(s) T_{4}(t) .
\end{aligned}
$$

For a proof of statements (i)-(iv) see Lemma 9. Statement (v) follows from $\Gamma(t+s)=\Gamma(t) \Gamma(s)$.

COROLLARY 13. Let $A$ be the generator of a strongly continuous semigroup on $E$ and let $B$ be a closed linear operator on $E$ with $D(A) \subset D(B)$. Then there is a $w \in \mathbf{R}$ such that for every $\lambda \in \mathbf{C}$ with $\operatorname{Re} \lambda>w, R_{\lambda}:=(\lambda(\lambda-A)-B)^{-1}$ exists. $R_{\lambda}$ is the Laplace transform of a strongly differentiable family $W(t)$ of bounded linear operators on $E$; i.e.,

$$
R_{\lambda} x=\int_{0}^{\infty} e^{-\lambda t} W(t) x d t
$$

for every $x \in E$ and every $\lambda \in \mathbf{C}$ with $\operatorname{Re} \lambda>w . W(t)$ is given as the inverse Laplace transform of $R_{\lambda}$; i.e.,

$$
W(t) x=\frac{1}{2 \pi i} \int_{\gamma} e^{\lambda t} R_{\lambda} x d \lambda
$$

for every $x \in E$. The family $(W(t))$ has the following properties:

(i) $W(0)=0$,

(ii) $W(\cdot): E \rightarrow D(A), B W(\cdot) x$ is continuous for every $x \in E, W^{\prime}(\cdot): D(A) \rightarrow$ $D(A)$ and $W^{\prime}(\cdot)$ is continuously differentiable for every $x \in D(A)$, 
(iii) $W^{\prime}(t) x=A W(t) x+\int_{0}^{t} B W(s) x d s+x$,

(iv) $W^{(2)}(t) x=A W^{\prime}(t) x+B W(t) x$.

The semigroup generated by $\Delta:=\left[\begin{array}{ll}A & I \\ B & 0\end{array}\right]$ is given by

$$
\left[\begin{array}{cc}
W^{\prime}(t) & W(t) \\
B W(t) & \int_{0}^{t} B W(s) d s+I
\end{array}\right] .
$$

PROOF. As we have seen in the proof of Proposition 7 the resolvent of $\Delta$ is given by

$$
\left[\begin{array}{cc}
\lambda R_{\lambda} & R_{\lambda} \\
B R_{\lambda} & (\lambda-A) R_{\lambda}
\end{array}\right]
$$

Define $W(t):=T_{2}(t)$ (see Lemma 12). Then the statements of the corollary follow from Lemma 12 and the fact that the Laplace transform of a strongly differentiable operator family is invertible for every $x \in E$.

COROLLARY 14. Let $A$ be the generator of a strongly continuous semigroup on $E$ and let $B$ be a closed linear operator on $E$ with $D(A) \subset D(B)$. Then, for every $x \in D(A)$ and $y \in E$, the integrated Cauchy problem

$$
u^{(1)}(t)=A u(t)+\int_{0}^{t} B u(s) d s+y, \quad u(0)=x
$$

has a unique solution $u(\cdot)$ in $C^{1}\left(\mathbf{R}^{+}, E\right)$. The solution is given by

$$
W^{\prime}(t) x+W(t) y=\frac{1}{2 \pi i} \int_{\gamma} e^{\lambda t} R_{\lambda}(\lambda x+y) d \lambda
$$

where $R_{\lambda}, W(t)$ are defined as in Corollary 13.

ProOF. Existence: Define

$$
\begin{aligned}
u(t) & :=W^{\prime}(t) x+W(t) y=\frac{1}{2 \pi i} \int_{\gamma} e^{\lambda t} R_{\lambda}(\lambda x+y) d \lambda \\
& =T_{1}(t) x+T_{2}(t) y
\end{aligned}
$$

$\left(T_{1}(t), T_{2}(t)\right.$ as in Lemma 12). Then $u(0)=x, B u(\cdot)$ is continuous and

$$
\begin{aligned}
u^{(1)}(t) & =A T_{1}(t) x+T_{3}(t) x+A T_{2}(t) y+T_{4}(t) y \\
& =A u(t)+\int_{0}^{t} B u(s) d s+y .
\end{aligned}
$$

Uniqueness. Let $v(\cdot)$ be a solution of the integrated problem with $x=y=0$; i.e., $v(\cdot): \mathbf{R}^{+} \rightarrow D(A)$ is in $C^{1}\left(\mathbf{R}^{+}, E\right), B v(\cdot)$ is integrable and

$$
v^{(1)}(t)=A v(t)+\int_{0}^{t} B v(s) d s, \quad v(0)=0 .
$$

Define $w(t):=\int_{0}^{t} v(s) d s$. Then

(a) $w(t) \in D(B)$ for every $t \geq 0$ and $w(0)=0$,

(b) $w^{\prime}(t) \in D(A)$ for every $t \geq 0$ and $w^{\prime}(0)=0$,

(c) $B w(\cdot)$ is continuous and

$$
w^{(2)}(t)-A w^{(1)}(t)-B w(t)=0 .
$$

Therefore, by Theorem $5, w(\cdot)=0$ or, by the continuity of $v(\cdot), v(\cdot)=0$ for every $t \geq 0$. Hence the solution of the integrated Cauchy problem is unique. 
THEOREM 15. Let $A$ be the generator of a strongly continuous semigroup on $E$ and let $B$ be a closed linear operator on $E$ with $D(A) \subset D(B)$. Let $f$ be a continuous function from $\mathbf{R}^{+}$into $E$ and let

$$
\left[\begin{array}{ll}
T_{1}(t) & T_{2}(t) \\
T_{3}(t) & T_{4}(t)
\end{array}\right]
$$

be the semigroup generated by $\Delta:=\left[\begin{array}{ll}A & I \\ B & 0\end{array}\right]$. Then the following statements are equivalent:

(i) $t \rightarrow \int_{0}^{t} T_{1}(t-s) f(s) d s$ is continuously differentiable.

(ii) The inhomogeneous damped second order Cauchy problem

$$
u^{(2)}(t)-A u^{(1)}(t)-B u(t)=f(t), \quad u(0)=x, \quad u^{(1)}(0)=y
$$

has for every $x, y \in D(A)$ a solution $u(\cdot)$ in $C^{2}\left(\mathbf{R}^{+}, E\right)$ such that $B u(\cdot)$ is continuous.

If $B R_{\lambda}=R_{\lambda} B$ on $D(A)$, then every solution is unique. Otherwise the solution is unique among all solutions $u(\cdot)$ for which $B u(\cdot)$ is a continuous function.

PROOF. (i) $\rightarrow$ (ii). Uniqueness: If there are solutions $u(\cdot)$ and $v(\cdot)$, then $h(\cdot):=$ $u(\cdot)-v(\cdot)$ is a solution of

$$
u^{(2)}(t)-A u^{(1)}(t)-B u(t)=0, \quad u(0)=u^{(1)}(0)=0 .
$$

If $B R_{\lambda}=R_{\lambda} B$ on $D(A)$ or if $B h(\cdot)$ is continuous, then, by Theorems 5 and 11, $h(\cdot)=0$. But then $u(\cdot)=v(\cdot)$.

Existence: Define

$$
\begin{aligned}
& x(t):=T_{1}(t) x+T_{2}(t)(y-A x)+\int_{0}^{t} T_{2}(t-s) f(s) d s, \\
& w(t):=\int_{0}^{t} T_{2}(t-s) f(s) d s .
\end{aligned}
$$

By Lemma 12 we obtain that $B x(\cdot)$ is a continuous function and

$$
\begin{aligned}
\frac{1}{h}(w(t+h)-w(t))= & \frac{1}{h}\left(T_{1}(h)-I\right) w(t) \\
& +\frac{1}{h} T_{2}(h) \int_{0}^{t} T_{4}(t-s) f(s) d s \\
& +\frac{1}{h} \int_{t}^{t+h} T_{2}(t+h-s) f(s) d s .
\end{aligned}
$$

From $A T_{2}(\cdot)=T_{4}(\cdot)-T_{1}(\cdot)$ we see that $A T_{2}(\cdot)$ is continuous and by the closedness of $A$ we get $w(\cdot): \mathbf{R}^{+} \rightarrow D(A)$. Therefore the right-hand side of the equation above converges as $h \rightarrow 0, x(\cdot)$ is continuously differentiable and

$$
x^{(1)}(t)=A x(t)+T_{3}(t) x+T_{4}(t)(y-A x)+\int_{0}^{t} T_{4}(t-s) f(s) d s .
$$

Now $x^{(1)}(\cdot)$ is continuously differentiable if and only if $A x(\cdot)$ is. But

$$
\begin{aligned}
A x(t)= & T_{2}(t) B x+T_{1}(t) y-T_{3}(t) x-T_{4}(t)(y-A x) \\
& -\int_{0}^{t} T_{4}(t-s) f(s) d s+\int_{0}^{t} T_{1}(t-s) f(s) d s
\end{aligned}
$$


and therefore $A x(\cdot)$ is continuously differentiable if and only if

$$
t \rightarrow \int_{0}^{t} T_{1}(t-s) f(s) d s
$$

is continuously differentiable. By (i) and the closedness of $A$ we obtain $(d / d t) A x(t)$ $=A x^{(1)}(t)$ and

$$
\begin{aligned}
x^{(2)}(t)= & A x^{(1)}(t)+T_{3}^{\prime}(t) x+T_{4}^{\prime}(t)(y-A x) \\
& +\frac{d}{d t} \int_{0}^{t} T_{4}(t-s) f(s) d s \\
= & A x^{(1)}(t)+B T_{1}(t) x+B T_{2}(t)(y-A x) \\
& +B \int_{0}^{t} T_{2}(t-s) f(s) d s+f(t) \\
= & A x^{(1)}(t)+B x(t)+f(t) .
\end{aligned}
$$

(ii) $\rightarrow$ (i). Let $x(\cdot)$ be a solution of the damped, inhomogeneous problem such that $B x(\cdot)$ is continuous. Then $A x(\cdot)$ is continuously differentiable. Define $u(\cdot), F(\cdot): \mathbf{R}^{+} \rightarrow E \times E$ by

$$
u(t):=\left(x(t), x^{(1)}(t)-A x(t)\right), \quad F(t):=(0, f(t)) .
$$

The function $u(\cdot)$ is continuously differentiable and

$$
u^{(1)}(t)=\Delta u(t)+F(t), \quad u(0)=(x, y-A x) .
$$

By [22, p. 106], we obtain

or

$$
u(t)=\Gamma(t)(x, y-A x)+\int_{0}^{t} \Gamma(t-s) F(s) d s
$$

$$
x(t)=T_{1}(t) x+T_{2}(t)(y-A x)+\int_{0}^{t} T_{2}(t-s) f(s) d s .
$$

But $x(\cdot)$ is twice continuously differentiable and, as seen in the first part of the proof, this is the case if and only if $t \rightarrow \int_{0}^{t} T_{1}(t-s) f(s) d s$ is continuously differentiable.

The easy proofs of the following lemmas are omitted.

LEMMA 16. Let $\{V(t), t \geq 0\}$ be a strongly continuous family of bounded operators on $E$ and let $f(\cdot)$ be an element of $C^{1}\left(\mathbf{R}^{+}, E\right)$. Then

$$
w(t):=\int_{0}^{t} V(t-s) f(s) d s
$$

is continuously differentiable and

$$
w^{(1)}(t)=\int_{0}^{t} V(t-s) f^{\prime}(s) d s+V(t) f(0) .
$$

LEMMA 17. Let $f(\cdot)$ be an integrable function and $\int_{0}^{t} T_{1}(t-s) f(s) d s \in D(A)$ for every $t \geq 0$. Then $t \rightarrow \int_{0}^{t} T_{1}(t-s) f(s) d s$ is continuously differentiable ( $A$ and $T_{1}(\cdot)$ are as in Theorem 15).

COROLLARY 18. Let $A$ be the generator of a strongly continuous semigroup on $E$ and let $B$ be a closed linear operator on $E$ with $D(A) \subset D(B)$. Let $f$ be a 
continuous function from $\mathbf{R}^{+}$into $E$ such that either

(i) $f(\cdot)$ is continuously differentiable or

(ii) $f(\cdot): \mathbf{R}^{+} \rightarrow D(A)$ and $A f(\cdot), B f(\cdot)$ are integrable.

Then, for every $x, y \in D(A)$, the inhomogeneous, damped second order Cauchy problem

$$
u^{(2)}(t)-A u^{(1)}(t)-B u(t)=f(t), \quad u(0)=x, \quad u^{(1)}(t)=y
$$

has a solution $u(\cdot)$ in $C^{2}\left(\mathbf{R}^{+}, E\right)$ given by

$$
W^{\prime}(t) x+W(t)(y-A x)+\int_{0}^{t} W(t-s) f(s) d s
$$

where $W(t)$ is defined as in Corollary 13. If $B R_{\lambda}=R_{\lambda} B$ on $D(A)$, then every solution is unique. Otherwise the solution is unique among all solutions $u(\cdot)$ for which $B u(\cdot)$ is a continuous function.

ProOF. (i) If $f(\cdot)$ is continuously differentiable, then, by Lemma $16, t \rightarrow$ $\int_{0}^{t} T_{1}(t-s) f(s) d s$ is continuously differentiable. Hence the statement of the corollary follows from Theorem 15.

(ii) Let $f(\cdot)$ be a function from $\mathbf{R}^{+}$into $D(A)$ such that $A f(\cdot)$ and $B f(\cdot)$ are integrable. From the equation

$$
A T_{1}(t-s) f(s)=T_{1}(t-s) A f(s)+T_{2}(t-s) B f(s)-T_{3}(t-s) f(s)
$$

we obtain $\int_{0}^{t} T_{1}(t-s) f(s) d s \in D(A)$ for every $t \geq 0$. By Lemma 17 and Theorem 15 the statement of the corollary follows.

Proposition 19. Let $A$ be the generator of a strongly continuous semigroup on $E$ and let $B$ be a closed linear operator on $E$ with $D(A) \subset D(B)$. Let $f$ be a continuous function from $\mathbf{R}^{+}$into $E$. Then, for every $x \in D(A)$, the integrated Cauchy problem

$$
u^{(1)}(t)=A u(t)+\int_{0}^{t}(B u(s)+f(s)) d s, \quad u(0)=x
$$

has a unique solution $u(\cdot)$ in $C^{1}\left(\mathbf{R}^{+}, E\right)$. The solution is given by

$$
W^{\prime}(t) x+\int_{0}^{t} W(t-s) f(s) d s
$$

where $W(t)$ is defined as in Corollary 13.

Proof. Define

$$
\begin{aligned}
x(t) & :=W^{\prime}(t) x+\int_{0}^{t} W(t-s) f(s) d s \\
& =T_{1}(t) x+\int_{0}^{t} T_{2}(t-s) f(s) d s .
\end{aligned}
$$

Then, as seen in the proof of Theorem $15, x(\cdot)$ is continuously differentiable, $x(t) \in$ $D(A)$ for every $t \geq 0$ and

$$
\begin{aligned}
x^{(1)}(t) & =A x(t)+T_{3}(t) x+\int_{0}^{t} T_{4}(t-s) f(s) d s \\
& =A x(t)+\int_{0}^{t}\left(B T_{1}(s) x+T_{4}(t-s) f(s)\right) d s .
\end{aligned}
$$


We have to show that

$$
\int_{0}^{t} T_{4}(t-s) f(s) d s=\int_{0}^{t}\left(B \int_{0}^{s} T_{2}(s-r) f(r) d r+f(s)\right) d s .
$$

Therefore define

$$
g(t):=\int_{0}^{t} T_{4}(t-s) f(s) d s
$$

and

$$
h(t):=\int_{0}^{t}\left(B \int_{0}^{s} T_{2}(s-r) f(r) d r+f(s)\right) d s .
$$

Then $g(0)=h(0)$ and

$$
h^{(1)}(t)=B \int_{0}^{t} T_{2}(t-r) f(r) d r+f(t) .
$$

Now, by Lemma 12,

$$
\begin{aligned}
\frac{1}{h}(g(t+h)-g(t))= & \frac{1}{h}\left(T_{4}(h)-I\right) g(t) \\
& +\frac{1}{h} T_{3}(h) \int_{0}^{t} T_{2}(t-s) f(s) d s \\
& +\frac{1}{h} \int_{t}^{t+h} T_{4}(t+h-s) f(s) d s .
\end{aligned}
$$

Therefore $g(\cdot)$ is continuously differentiable and

$$
g^{(1)}(t)=B \int_{0}^{t} T_{2}(t-s) f(s) d s+f(t)=h^{(1)}(t) .
$$

This proves that $x(\cdot)$ is a solution of the integrated problem. Furthermore

$$
\begin{aligned}
B x(t) & =B T_{1}(t) x+\int_{0}^{t} B T_{2}(t-s) f(s) d s \\
& =T_{3}^{\prime}(t) x+\int_{0}^{t} T_{3}(t-s) f(s) d s
\end{aligned}
$$

Hence $B x(\cdot)$ is even continuous and not only integrable. Suppose there is another solution $v(\cdot)$ of the integrated problem. Then $h(\cdot)=x(\cdot)-v(\cdot)$ is a solution of

$$
u^{(1)}(t)=A u(t)+\int_{0}^{t} B u(s) d s, \quad u(0)=0 .
$$

By Corollary 14 it follows that $h(t)=0$ for every $t \geq 0$.

In the same way as in Theorem 15 we characterize existence of solutions of a semilinear damped second order equation.

PROPOSITION 20. Let $A$ be the generator of a strongly continuous semigroup on $E$ and let $B$ be a closed linear operator on $E$ with $D(A) \subset D(B)$. Let $f$ be $a$ function from $[0, T] \times E$ into $E$ such that $f(\cdot, u(\cdot))$ is a continuous function from $[0, T]$ into $E$ whenever $u(\cdot) \in C([0, T], E)$. Define $W(t)\left(=T_{2}(t)\right)$ as in Corollary 
14 (as in Lemma 12). The following statements are equivalent:

(i) For every $x, y \in D(A)$ the semilinear damped Cauchy problem

$$
u^{(2)}(t)-A u^{(1)}(t)-B u(t)=f(t, u(t)), \quad u(0)=x, \quad u^{(1)}(0)=y
$$

has a solution $u(\cdot)$ in $C^{2}([0, T], E) \cap C^{1}([0, T],[D(A)])$.

(ii) For every $x, y \in D(A)$ the integral equation

$$
u(t)=W^{\prime}(t) x+W(t)(y-A x)+\int_{0}^{t} W(t-s) f(s, u(s)) d s
$$

has a solution $u(\cdot)$ in $C([0, T], E)$ such that $t \rightarrow \int_{0}^{t} W^{\prime}(t-s) f(s, u(s)) d s$ is continuously differentiable on $[0, T]$.

Proof. (i) $\rightarrow$ (ii). Let $u(\cdot)$ be in $C^{2}([0, T], E) \cap C^{1}([0, T],[D(A)])$. Then $u^{(1)}(\cdot)$ maps $[0, T]$ into $D(A)$ and $A u(\cdot)$ is continuously differentiable. Let $\Delta:=\left[\begin{array}{ll}A & I \\ B & 0\end{array}\right]$. Define

$$
x(t)=\left(x_{1}(t), x_{2}(t)\right):=\left(u(t), u^{(1)}(t)-A u(t)\right)
$$

and $F:[0, T] \times(E \times E) \rightarrow E \times E, F(t, x(t)):=\left(0, f\left(t, x_{1}(t)\right)\right.$. Then $x(\cdot):[0, T] \rightarrow$ $D(\Delta), x(\cdot) \in C^{1}([0, T], E \times E)$ and

$$
x^{(1)}(t)=\Delta x(t)+F(t, x(t)), \quad x(0)=(x, y-A x) .
$$

Therefore $x(\cdot)$ is a continuously differentiable solution of the integral equation

$$
u(t)=\Gamma(t) u(0)+\int_{0}^{t} \Gamma(t-s) F(s, u(s)) d s
$$

where $\Gamma(t)$ is the semigroup generated by $\Delta$ (see Lemma 12). This implies that $x_{1}(\cdot) \in C^{1}([0, T], E)$ is a solution of

$$
\begin{aligned}
u(t) & =T_{1}(t) x+T_{2}(t)(y-A x)+\int_{0}^{t} T_{2}(t-s) f(s, u(s)) d s \\
& =W^{\prime}(t) x+W(t)(y-A x)+\int_{0}^{t} W(t-s) f(s, u(s)) d s
\end{aligned}
$$

By definition of $x_{1}(\cdot)$ we have $x_{1}(\cdot) \in C^{2}([0, T], E)$. In the same way as in the proof of Theorem 15 we obtain

$$
\begin{aligned}
x_{1}^{(1)}(t) & =T_{2}(t) B x+T_{1}(t) y+\int_{0}^{t} T_{1}(t-s) f\left(s, x_{1}(s)\right) d s \\
& =W(t) B x+W^{\prime}(t) y+\int_{0}^{t} W^{\prime}(t-s) f\left(s, x_{1}(s)\right) d s .
\end{aligned}
$$

From the continuous differentiability of $x_{1}^{(1)}(\cdot), W(\cdot) B x$ and $W^{\prime}(\cdot) y$ we conclude that $t \rightarrow \int_{0}^{t} W^{\prime}(t-s) f(s, u(s)) d s$ is continuously differentiable on $[0, T]$.

The proof of (ii) $\rightarrow$ (i) is the same as the corresponding part of the proof of Theorem 15 and is omitted.

COROLLARY 21. Let $A$ be the generator of a strongly continuous semigroup on $E$ and let $B$ be a closed linear operator on $E$ with $D(A) \subset D(B)$. Let $f$ be $a$ function from $[0, T] \times E$ into $E$ such that $f(\cdot, u(\cdot))$ is a continuous function from $[0, T]$ into $E$ whenever $u(\cdot) \in C([0, T], E)$. Define $W(t)\left(=T_{2}(t)\right)$ as in Corollary 
14 (as in Lemma 12) and let $x \in D(A)$. The following statements are equivalent:

(i) The integral equation

$$
u(t)=W^{\prime}(t) x+\int_{0}^{t} W(t-s) f(s, u(s)) d s
$$

has a solution $v(\cdot)$ in $C([0, T], E)$.

(ii) The integrated Cauchy problem

$$
u^{(1)}(t)=A u(t)+\int_{0}^{t}(B u(s)+f(s, u(s)) d s, \quad u(0)=x
$$

has a solution $v(\cdot)$ with the properties $v(\cdot):[0, T] \rightarrow D(A), v(\cdot) \in C^{1}([0, T], E)$ and $B v(\cdot) \in C([0, T], E)$.

PROOF. (i) $\rightarrow$ (ii). Let $v(\cdot)$ be a solution of the integral equation; i.e.,

$$
v(t)=T_{1}(t) x+\int_{0}^{t} T_{2}(t-s) f(s, v(s)) d s .
$$

Then $v(\cdot):[0, T] \rightarrow D(A), v(\cdot) \in C^{1}([0, T], E)$ and

$$
\begin{aligned}
v^{(1)}(t) & =A v(t)+T_{3}(t) x+\int_{0}^{t} T_{4}(t-s) f(s, v(s)) d s \\
& =A v(t)+\int_{0}^{t} B T_{1}(s) x+T_{4}(t-s) f(s, v(s)) d s .
\end{aligned}
$$

In the same way as in the proof of Proposition 19 we show

$$
T_{4}(t-s) f(s, v(s))=B \int_{0}^{s} T_{2}(s-r) f(r, v(r)) d r+f(s, v(s)) .
$$

Therefore $v(\cdot)$ is a solution of the integrated Cauchy problem and $B v(\cdot)=T_{3}^{\prime}(t) x+$ $\int_{0}^{t} T_{3}(t-s) f(s, v(s)) d s$ is a continuous function.

(ii) $\rightarrow$ (i). Let $v(\cdot)$ be a solution of the integrated Cauchy problem with the properties stated in (ii). Then the function

$$
t \rightarrow x(t):=\left(v(t), \int_{0}^{t} B v(s)+f(s, v(s)) d s\right)
$$

is in $C^{1}([0, T], E \times E), x(\cdot):[0, T] \rightarrow D(\Delta)\left(\Delta=\left[\begin{array}{cc}A & I \\ B & 0\end{array}\right]\right)$ and

$$
\Delta x(t)=\left(v^{(1)}(t), B v(t)\right)=x^{(1)}(t)-F(t, x(t))
$$

where $F(t, x(t)):=\left(0 ; f\left(t, x_{1}(t)\right)\right.$ and $x(t):=\left(x_{1}(t), x_{2}(t)\right)$. Therefore $x(\cdot)$ is a solution of $x^{(1)}(t)=\Delta x(t)+F(t, x(t)), x(0)=(x, 0)$, and hence a solution of

$$
x(t)=\Gamma(t)(x, 0)+\int_{0}^{t} \Gamma(t-s) F(s, x(s)) d s
$$

where $\Gamma(t)$ is the semigroup generated by $\Delta$. This implies

$$
x_{1}(t)=T_{1}(t) x+\int_{0}^{t} T_{2}(t-s) f\left(s, x_{1}(s)\right) d s .
$$

But $x_{1}(t)=v(t)$ and therefore $v(\cdot)$ is a solution of the integral equation. 
EXAMPLE. Let $A$ be the generator of a strongly continuous semigroup $(T(t))$ on a Banach space. In many applications (e.g. the linear strongly damped wave equation, the Klein-Gordon equation, the vibrating beam equation etc., see the discussion following Corollary 10, [1 and 27]) the damped second order Cauchy problem appears in the form

$$
\begin{aligned}
& u^{(2)}(t)-(a A+b I) u^{(1)}(t)-(c A+d I) u(t)=f(t), \\
& u(0)=x, \quad u^{(1)}(0)=y
\end{aligned}
$$

where $a$ is a positive real number, $b, c, d$ are arbitrary complex numbers and $f(\cdot)$ is a function satisfying, for example, the conditions of Corollary 18. As proved in Theorem 11, the homogeneous Cauchy problem (\#) is well-posed. By Corollary 18 , for every $x, y \in D(A)$ the unique solution of $(\#)$ is given by

$$
W^{\prime}(t) x+W(t)(y-A x)+\int_{0}^{t} W(t-s) f(s) d s
$$

where $W(t)$ is the inverse Laplace transform of

$$
\begin{aligned}
\lambda \rightarrow R_{\lambda} & =\left(\lambda(\lambda-(a A+b I)-(c A+d I))^{-1}\right. \\
& =\frac{1}{a \lambda+c} R\left(\frac{1}{a \lambda+c}\left(\lambda^{2}-\lambda b-d\right), A\right) .
\end{aligned}
$$

In order to determine $W(t)$ we will recall two elementary operations from Laplace transform theory (see [4]):

(1) If $F(t)$ is the inverse Laplace transform of $f(\lambda)$, then $(1 / a) e^{b t / a} F(t / a)$ is the inverse Laplace transform of $f(a \lambda-b)$ if $a>0$ and $b \in \mathbf{C}$.

(2) If $F(t)$ is the inverse Laplace transform of $f(\lambda)$, then

$$
\int_{0}^{t} J_{0}(2 \sqrt{(t-s) s}) F(s) d s
$$

is the inverse Laplace transform of $(1 / \lambda) f(\lambda+1 / \lambda)$, where $J_{0}(t)$ stands for the Bessel function

$$
\sum_{n=0}^{\infty}(-1)^{n} \frac{1}{(n !)^{2}}\left(\frac{t}{2}\right)^{2 n}
$$

Now suppose $q:=\left(-d+c^{2} / a^{2}+b c / a\right)^{-1 / 2}>0$. Define $f:=1 / a q, g:=f / q$, $h:=(1 / a)(b+2 c / a)$ and $i:=h / f$. Then, by the elementary operations (1) and (2) the following is true:

(a) $(q / a) R(\lambda, A) x$ is the Laplace transform of $(q / a) T(t) x$.

(b) $(q / a) R(f(\lambda+i), A) x$ is the Laplace transform of $(q / a f) e^{-i t} T(t / f) x$.

(c) $(q / a) \cdot(1 / \lambda) R(f(\lambda+1 / \lambda+i), A) x$ is the Laplace transform of

$$
\int_{0}^{t} J_{0}(2 \sqrt{(t-s)-s}) \frac{q}{a f} e^{-i s} T\left(\frac{s}{f}\right) x d s .
$$

(d)

$$
\frac{1}{a} \cdot \frac{1}{\lambda} R\left(f\left(q \lambda+\frac{1}{q \lambda}+i\right), A\right) x=\frac{1}{a} \cdot \frac{1}{\lambda} R\left(\frac{1}{a} \lambda+\frac{q}{\lambda}+h, A\right) x
$$


is the Laplace transform of

$$
\frac{1}{a f} \int_{0}^{t / q} J_{0}\left(2 \sqrt{\left(\frac{t}{q}-s\right) s}\right) e^{-i s} T\left(\frac{s}{f}\right) x d s .
$$

(e)

$$
\begin{gathered}
\frac{1}{a} \cdot \frac{1}{\lambda+c / a} R\left(\frac{1}{a}\left(\lambda+\frac{c}{a}\right)+\frac{q}{\lambda+c / a}+h, A\right) x \\
=\frac{1}{a \lambda+c} R\left(\frac{1}{a \lambda+c}\left(\lambda^{2}-\lambda b-d\right), A\right)
\end{gathered}
$$

is the Laplace transform of

$$
\begin{aligned}
& \frac{1}{a f} e^{-(c / a) t} \int_{0}^{t / q} J_{0}\left(2 \sqrt{\left(\frac{t}{q}-s\right) s}\right) e^{-i s} T\left(\frac{s}{f}\right) x d s \\
& =e^{-(c / a) t} \int_{0}^{t} J_{0}\left(\frac{2}{q} \sqrt{(t-s) s}\right) e^{-a h s} T(a s) x d s \\
& =e^{-(c / a) t} \int_{0}^{t} J_{0}\left[2 \sqrt{-d+\frac{c^{2}}{a^{2}}+\frac{b c}{a}} \cdot \sqrt{(t-s) s}\right] e^{(b+2(c / a)) s} T(a s) x d s
\end{aligned}
$$

Therefore, for $q>0$ and every $x \in E, W(t) x$ is given by the last expression. By some tedious calculations it can be seen that this expression is a solution of (\#) (with $v(0)=0$ and $v^{\prime}(0)=x$ ) not only for $q>0$ but for every complex number $b, c, d$ and every $a>0$. Hence, by Theorem $11, W(t) x$ is given by

$$
e^{-(c / a) t} \int_{0}^{t} J_{0}\left[2 \sqrt{-d+\frac{c^{2}}{a^{2}}+\frac{b c}{a}} \cdot \sqrt{(t-s) s}\right] e^{(b+2(c / a)) s} T(a s) x d s .
$$

If the semigroup $(T(t))$ generated by $A$ is known we obtain by

$$
W^{\prime}(t) x+W(t)(y-A x)+\int_{0}^{t} W(t-s) f(s) d s
$$

an explicit expression of the unique solution of (\#).

ACKNOWLEDGEMENT. I wish to acknowledge my appreciation of my thesis adviser, Professor Rainer Nagel, for his guidance and suggestions in the preparation of this dissertation. Also, the author would like to express his gratitude to the "Arbeitsgruppe Funktionalanalysis" at the University of Tuebingen for many interesting discussions and to Professor Jerome A. Goldstein for his hospitality and encouragement on the occasion of a research visit at Tulane University, New Orleans.

\section{REFERENCES}

1. P. Aviles and J. T. Sandefur, A new approach to nonlinear second order equations with applications to partial differential equations, J. Differential Equations 58 (1985), 404-427.

2. E. Davies, One-parameter semigroups, Academic Press, New York, 1980.

3. G. Da Prato and E. Giusti, Una caratterizzazione dei generatori di funzioni coseno astratte, Boll. Un. Mat. Ital. 22 (1967), 357-362.

4. G. Doetsch, Handbuch der Laplace Transformationen, Band I, Birkhäuser Verlag, Basel, 1950. 
5. H. O. Fattorini, Un teorema de perturbación para generadores de funciones coseno, Rev. Un. Mat. Argentina 25 (1971), 200-211.

6. __ The abstract Cauchy problem, Addison-Wesley, Reading, Mass., 1983.

7. ___ Ordinary differential equations in linear topological spaces. I, J. Differential Equation 5 (1969), 72-105.

8. __ Ordinary differential equations in linear topological spaces. II, J. Differential Equations 6 (1969), 50-70.

9. Extension and behavior at infinity of solutions of certain linear operational differential equations, Pacific J. Math. 33 (1970), 583-615.

10. __ Some remarks on second order abstract Cauchy problems, Funkc. Ekvac. 24 (1981), 331-344.

11. W. E. Fitzgibbon, Strongly damped quasilinear evolution equations, J. Math. Anal. Appl. 79 (1981), 536-550.

12. J. A. Goldstein, Semigroups of linear operators and applications, Oxford Univ. Press, New York, 1985.

13. E. Hille, Une généralisation du problème de Cauchy, Ann. Inst. Fourier (Grenoble) 4 (1952), 31-48.

14. E. Hille and R. S. Phillips, Functional analysis and semigroups, Amer. Math. Soc. Colloq. Publ., vol. 31, Providence, R.I., 1957.

15. J. Kisyński, On cosine operator functions and one-parameter groups of operators, Studia Math. 44 (1973), 93-105.

16. S. G. Krein, Linear differential equations in Banach space, Amer. Math. Soc., Providence, R.I., 1971.

17. D. Lutz, Strongly continuous operator cosine functions, Functional Analysis (Proc. Dubrovnik, 1981), Lecture Notes in Math., vol. 948, Springer-Verlag, Berlin and New York, 1982, pp. 73-97.

18. P. Massat, Limiting behavior for strongly damped nonlinear wave equations, J. Differential Equations 48 (1983), 334-349.

19. B. Nagy, Cosine operator functions and the abstract Cauchy problem, Period. Math. Hungar. 7 (1976), 213-217.

20. 281-289.

21. F. Neubrander, Well-posedness of abstract Cauchy problems, Semigroup Forum 29 (1984), 75-85.

22. A. Pazy, Semigroups of linear operators and applications to partial differential equations, Springer-Verlag, New York and Berlin, 1983.

23. R. S. Phillips, A note on the abstract Cauchy problem, Proc. Nat. Acad. Sci. U.S.A. 40 (1954), 244-248.

24. , Perturbation theory for semigroups of linear operators, Trans. Amer. Math. Soc. 74 (1953), 199-221.

25. J. T. Sandefur, Higher order abstract Cauchy problems, Dissertation, Tulane University, New Orleans, 1974

26. __ Higher order abstract Cauchy problems, J. Math. Anal. Appl. 60 (1977), 228-248.

27. __ Existence and uniqueness of solutions of second order nonlinear differential equations, Siam J. Math. Anal. 14 (1983), 477-487.

28. M. Sova, Cosine operator functions, Rozprawy Math. 49 (1966), 1-46.

29. C. C. Travis and G. F. Webb, Cosine families and abstraci nonlinear second order differential equations, Acta Math. Acad. Sci. Hungar. 32 (1978), 75-96.

30. M. Watanabe, A peturbation theory for abstract evolution equations of second order, Proc. Japan Acad. Ser. A 58 (1982), 143-146.

31. G. F. Webb, Existence and asymptotic behavior for a strongly damped nonlinear wave equation, Canad. J. Math. 32 (1980), 631-643.

32. B. Weiss, Abstract vibrating systems, J. Math. Mech. 17 (1967), 241-255.

33. D. V. Widder, The Laplace transform, Princeton Univer. Press, Princeton, N.J., 1946.

Department of Mathematics, Georgetown University, Washington, D.C. 